\title{
LIFTING CLASSES FOR THE FIXED POINT THEORY OF $n$-VALUED MAPS
}

\author{
Robert F. Brown \\ Department of Mathematics, University of California, Los Angeles, CA 90095-1555 \\ e-mail: rfb@math.ucla.edu \\ Charlotte Deconinck, Karel Dekimpe* \\ KU Leuven Campus Kulak Kortrijk, 8500 Kortrijk, Belgium \\ e-mail: Charlotte.Deconinck@kuleuven.be, Karel.Dekimpe@kuleuven.be \\ P. Christopher Staecker \\ Department of Mathematics, Fairfield University, Fairfield, CT 06824 \\ e-mail: cstaecker@fairfield.edu
}

October 3, 2019

\begin{abstract}
The theory of lifting classes and the Reidemeister number of singlevalued maps of a finite polyhedron $X$ is extended to $n$-valued maps by replacing liftings to universal covering spaces by liftings with codomain an orbit configuration space, a structure recently introduced by Xicoténcatl. The liftings of an $n$-valued map $f$ split into self-maps of the universal covering space of $X$ that we call lift-factors. An equivalence relation is defined on the lift-factors of $f$ and the number of equivalence classes is the Reidemeister number of $f$. The fixed point classes of $f$ are the projections of the fixed point sets of the lift-factors and are the same as those of Schirmer. An equivalence relation is defined on the fundamental group of $X$ such that the number of equivalence classes equals the Reidemeister number. We prove that if $X$ is a manifold of dimension at least three, then algebraically the orbit configuration space approach is the same as one utilizing the universal covering space. The Jiang subgroup is extended to $n$-valued maps as a subgroup of the group of covering transformations of the orbit configuration space and used to find conditions under which the Nielsen number of an $n$-valued map equals its Reidemeister number. If an $n$-valued map splits into $n$ single-valued maps, then its $n$-valued Reidemeister number is the sum of their Reidemeister numbers.
\end{abstract}

Keywords and Phrases: lifting, $n$-valued map, Reidemeister number, Nielsen number, configuration space, universal covering space,

\footnotetext{
${ }^{*}$ Research supported by long term structural funding - Methusalem grant of the Flemish Government.
} 
Jiang subgroup, orbit configuration space, semidirect product, fixed point class, cyclic homotopy, braid group, lift-factor

Subject Classification: 55M20, 54C60, 57M10, 55R80, 57M05

\section{Introduction}

Throughout the paper, the space $X$ will be a connected finite polyhedron. Given some natural number $n>0$, a set-valued function $f: X \multimap X$ is an $n$-valued map if it is continuous, that is, both upper and lower semi-continuous, and the cardinality of $f(x)$ is exactly $n$ for each $x$, see 3 .

For the Nielsen fixed point theory of a single-valued map $f: X \rightarrow$ $X$, the set of liftings $\tilde{f}: \tilde{X} \rightarrow \tilde{X}$ to the universal covering space $p: \tilde{X} \rightarrow X$ is partitioned into equivalence classes under conjugation by covering transformations. An equivalence class is called a lifting class and the number of such classes, which may be infinite, is the Reidemeister number $R(f)$ of the map $f$ [12], [13]. The fixed point sets $\operatorname{Fix}(\tilde{f})$ of equivalent liftings, if nonempty, are mapped by $p$ to the same subsets of $\operatorname{Fix}(f)$. The sets $p \operatorname{Fix}(\tilde{f})$ are the fixed point classes of the map $f$ and the number of such classes of nonzero fixed point index, called the Nielsen number, is a lower bound for the number of fixed points of every map homotopic to $f$.

The purpose of this paper is to extend the theory of lifting classes to the setting of $n$-valued maps. In order to do so, following [8] we will view an $n$-valued map as a single-valued map from $X$ to a space of subsets of $X$. Let $F_{n}(X)$ be the configuration space of $n$ ordered points on $X$, defined as:

$$
F_{n}(X)=\left\{\left(x_{1}, \ldots, x_{n}\right) \mid i \neq j \text { implies } x_{i} \neq x_{j}\right\} .
$$

which is topologized as a subset of the $n$-fold Cartesian product of $X$. Let $D_{n}(X)$ be the configuration space of $n$ unordered points on $X$, defined as:

$$
D_{n}(X)=\left\{\left\{x_{1}, \ldots, x_{n}\right\} \mid i \neq j \text { implies } x_{i} \neq x_{j}\right\} .
$$

Thus $D_{n}(X)$ is the orbit space of $F_{n}(X)$ under the free action of the symmetric group $\Sigma_{n}$ and the quotient map $q: F_{n}(X) \rightarrow D_{n}(X)$, which induces the quotient topology on $D_{n}(X)$, is a covering space of order $n$ !. We will not distinguish between an $n$-valued map $f: X \multimap$ $X$, and the corresponding function $f: X \rightarrow D_{n}(X)$, which is also continuous [3]. Thus we may refer to a map $f: X \rightarrow D_{n}(X)$ as an $n$-valued map. 
As we will discuss in Section 2, the lifting classes for $f: X \rightarrow$ $D_{n}(X)$ will not be classes of maps of the corresponding universal covering spaces because if $n>1$ then such a lifting does not have a well-defined fixed point set. Instead we will consider liftings $\bar{f}$ of $f$ from the universal covering space $\tilde{X}$ to an intermediate covering space, the orbit configuration space $p^{n}: F_{n}(\tilde{X}, \pi) \rightarrow D_{n}(X)$ where

$F_{n}(\tilde{X}, \pi)=\left\{\left(\tilde{x}_{1}, \ldots, \tilde{x}_{n}\right) \mid i \neq j\right.$ implies $\tilde{x}_{i} \neq \alpha \tilde{x}_{j}$ for all $\left.\alpha \in \pi_{1}(X)\right\}$.

Since $F_{n}(\tilde{X}, \pi)$ is a subspace of $F_{n}(\tilde{X})$, we may write a lifting in the form $\bar{f}=\left(\bar{f}_{1}, \bar{f}_{2}, \ldots, \bar{f}_{n}\right)$ where each $\bar{f}_{i}$, called a lift-factor, is a map of $\tilde{X}$ to itself. Conjugation by covering transformations partitions the set of all lift-factors of all liftings of $f$ into equivalence classes. In the setting of $n$-valued maps, the Reidemeister number $R(f)$ is the number of equivalence classes of lift-factors. The fixed point set of $f$ is the union of the images of the fixed point sets of the lift-factors, which are defined to be the fixed point classes of $f$. We prove that these images are identical if the lift-factors are equivalent and disjoint otherwise. Therefore the number of fixed point classes equals the Reidemeister number.

Also in Section 2, choosing appropriate base points, we call the lifting $\bar{f}^{*}$ of $f$ that preserves the base points its basic lifting. Then, writing $\bar{f}^{*}=\left(\bar{f}_{1}^{*}, \bar{f}_{2}^{*}, \ldots, \bar{f}_{n}^{*}\right)$, every lift-factor of $f$ can be written in the form $\alpha \bar{f}_{i}^{*}$ for some $\alpha \in \pi_{1}(X)$ and $1 \leq i \leq n$. As a tool for the subsequent calculations of the Reidemeister number, we use the basic lifting to define a homomorphism $\psi_{f}: \pi_{1}(X) \rightarrow \pi_{1}(X)^{n} \rtimes \Sigma_{n}$, where this semi-direct product of the $n$-fold direct product of $\pi_{1}(X)$ and the symmetric group of order $n$ is isomorphic to the group of covering transformations of the orbit configuration space, by setting $\psi_{f}(\gamma) \circ \bar{f}^{*}=\bar{f}^{*} \circ \gamma$. We then can write $\psi_{f}(\gamma)=\left(\phi_{1}(\gamma), \ldots, \phi_{n}(\gamma) ; \sigma_{\gamma}\right)$ where $\phi_{i}: \pi_{1}(X) \rightarrow \pi_{1}(X)$ and $\sigma: \pi_{1}(X) \rightarrow \Sigma_{n}$ with $\sigma_{\gamma}=\sigma(\gamma)$.

Helga Schirmer, in initiating the Nielsen fixed point theory for $n$-valued maps in [14], extended the classical definition of the fixed point classes to $n$-valued maps. As a model for her definition, she did not use images of fixed point sets of liftings but, instead, an equivalent definition in terms of paths in the space. Gert-Jan Dugardein reformulated Schirmer's theory in terms of a definition of lifting classes different than the one we introduce in Section 2, but one that is equivalent to it, and he showed that the fixed point classes defined as images of the fixed point sets of those liftings are the same as the classes defined by Schirmer. We will present Dugardein's results in Section 3 and demonstrate that our definition of the fixed point classes is equivalent to Schirmer's. 
In the fixed point theory of a single-valued map $f: X \rightarrow X$, there is a twisted conjugacy relation defined on the fundamental group $\pi_{1}(X)$ of $X$ by setting $\alpha$ equivalent to $\beta$ if there exists $\gamma \in \pi_{1}(X)$ such that $\alpha=\gamma \beta f_{\pi}\left(\gamma^{-1}\right)$ where $f_{\pi}$ is the fundamental group homomorphism induced by $f$. The equivalence classes are in oneto-one correspondence with the lifting classes and thus the Reidemeister number is the number of equivalence classes with respect to this twisted conjugacy. In the setting of $n$-valued maps, for $n>1$ the approach in Section 4 is somewhat different. We utilize the homomorphism $\psi_{f}: \pi_{1}(X) \rightarrow \pi_{1}(X)^{n} \rtimes \Sigma_{n}$ where $\psi_{f}(\gamma)=$ $\left(\phi_{1}(\gamma), \ldots, \phi_{n}(\gamma) ; \sigma_{\gamma}\right)$ that we introduced in Section 2. We define an equivalence relation $\sim_{i}$ on $\pi_{1}(X)$ by setting $\alpha \sim_{i} \beta$ if there exists $\gamma \in \pi_{1}(X)$ such that $\sigma_{\gamma}(i)=i$ and $\alpha=\gamma \beta \phi_{i}\left(\gamma^{-1}\right)$. This is not a twisted conjugacy relation because $\phi_{i}$ is in general not a homomorphism. However, the Reidemeister number is then the number of equivalence classes. We illustrate this approach by computing the Reidemeister number of a specific 3-valued map of the 2-torus.

In Section 5 we calculate the Reidemeister number for all $n$ valued maps $f: S^{1} \multimap S^{1}$ of the circle. Viewing the circle as the complex numbers of norm one, it was proved in [2] that every such map is $n$-valued homotopic to a map that takes $z$ to the set of $n$-th roots of $z^{d}$ for some integer $d$. We use the results of the previous section to prove that $R(f)=|n-d|$ if $d \neq n$ and $R(f)=\infty$ if $d=n$.

Since the Reidemeister number of a single-valued map $f: X \rightarrow X$ is determined by a twisted conjugacy relation that depends on the induced fundamental group homomorphism $f_{\pi}: \pi_{1}(X) \rightarrow \pi_{1}(X)$, it would be natural to define the Reidemeister number of an $n$-valued map $f: X \rightarrow D_{n}(X)$ in terms of the induced fundamental group homomorphism $f_{\pi}: \pi_{1}(X) \rightarrow \pi_{1}\left(D_{n}(X)\right)$. Although, for reasons explained above, we have utilized the orbit configuration space of $\tilde{X}$ in place of the universal covering space of $D_{n}(X)$, in Section 6 we prove that if $X$ is a manifold of dimension at least three, then algebraically the two approaches are the same. A notable feature of this section is a demonstration that the configuration space $F_{n}(X)$ is connected (if $X$ is a connected polyhedron not homeomorphic to the interval or circle) that is modeled on an argument regarding robot motion planning.

For a map $f: X \rightarrow X$, Jiang in [1] introduced a subgroup $J(\tilde{f})$ of the fundamental group that is called the Jiang subgroup of the map $f$. It consists of the elements $\alpha \in \pi_{1}(X)$ such that there is a cyclic homotopy $H: X \times I \rightarrow X$, that is, a homotopy with the property 
that $H(x, 0)=H(x, 1)=f(x)$ that, when lifted to the universal covering space, induces a homotopy between $\tilde{f}$ and $\alpha \tilde{f}$, where $\alpha \in$ $\pi_{1}(X)$ is identified with the corresponding covering transformation. In Section 7 we extend Jiang's theory to $n$-valued maps. Lifting a cyclic homotopy $H: X \times I \rightarrow D_{n}(X)$ to a homotopy starting at the basic lifting $\bar{f}^{*}: \tilde{X} \rightarrow F_{n}(\tilde{X}, \pi)$ determines an element of $\pi(X)^{n} \rtimes$ $\Sigma_{n}$ at the other end of the lifting and thus the cyclic homotopies determine a subgroup $J_{n}\left(\bar{f}^{*}\right)$ of $\pi(X)^{n} \rtimes \Sigma_{n}$, the Jiang subgroup for $n$-valued maps. If $\psi_{f}\left(\pi_{1}(X)\right) \subseteq J_{n}\left(\bar{f}^{*}\right)$, where $\psi_{f}: \pi_{1}(X) \rightarrow$ $\pi_{1}(X)^{n} \rtimes \Sigma_{n}$ is the homomorphism introduced in Section 2, and for each $i \in\{1, \ldots, n\}$, there exists $\gamma \in \pi(X)$ such that $\sigma_{\gamma}(j)=i$ for some $j \in\{1, \ldots, n\}$ then all the fixed point classes of $f$ have the same fixed point index and thus the Nielsen number $N(f)$ has the property that either $N(f)=0$ or $N(f)=R(f)$. If $X=T^{q}$ is the $q$-torus for $q \geq 1$, then $\psi_{f}\left(\pi_{1}\left(T^{q}\right)\right) \subseteq J_{n}\left(\bar{f}^{*}\right)$ for all $n$-valued maps. In particular, if $f_{n, A}: T^{q} \rightarrow D_{n}\left(T^{q}\right)$ is a linear $n$-valued map of [4], then either $N\left(f_{n, A}\right)=0$ or $N\left(f_{n, A}\right)=R\left(f_{n, A}\right)$.

A final section discusses split n-valued maps, that is, maps $f: X \rightarrow$ $D_{n}(X)$ for which there exist single-valued maps $f_{1}, \ldots, f_{n}: X \rightarrow X$ such that $f(x)=\left\{f_{1}(x), \ldots, f_{n}(x)\right\}$ for all $x \in X$. We prove that in this case its Reidemeister number for $n$-valued maps is the sum of the Reidemeister numbers of the $f_{i}$.

We thank the referee for helpful comments and suggestions.

\section{Coverings of $D_{n}(X)$}

Let $X$ be a space such that $F_{n}(X)$ is connected and let $u: \tilde{F}_{n}(X) \rightarrow$ $F_{n}(X)$ be the universal covering space. There is a covering $q$ : $F_{n}(X) \rightarrow D_{n}(X)$ so, since $\tilde{F}_{n}(X)$ is a universal cover, it is simply connected and thus $\tilde{F}_{n}(X)$ is the universal covering space of $D_{n}(X)$ with covering projection $q u: \tilde{F}_{n}(X) \rightarrow D_{n}(X)$.

Let $p: \tilde{X} \rightarrow X$ be the universal covering space of $X$. A map $f: X \rightarrow D_{n}(X)$ has a lifting $\tilde{f}$ to the universal covering spaces:

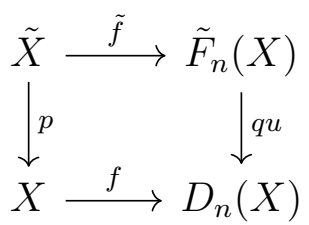

But, in contrast to the setting of single-valued maps, the lifting of an $n$-valued map $f: X \rightarrow D_{n}(X)$ to the universal covering spaces, for $n>1$, is not a convenient object of study because the fixed point 
set of $\tilde{f}$ is not defined. Consequently, the map $f$ will not be lifted to the universal covering spaces, but instead we will make use of an intermediate covering.

Let $E$ be a space and $G$ be a group acting on $E$ such that the projection $E \rightarrow E / G$ is a principal fibration. Then Xicoténcatl defined the orbit configuration space of $n$ ordered points (see [16]) as:

$$
F_{n}(E, G)=\left\{\left(e_{1}, \ldots, e_{n}\right) \in F_{n}(E) \mid G e_{i} \neq G e_{j} \text { for } i \neq j\right\} .
$$

We will make use of the orbit configuration space $F_{n}\left(\tilde{X}, \pi_{1}(X)\right)$ which we will write more compactly as $F_{n}(\tilde{X}, \pi)$. In Section [6 we will show that both $F_{n}(X)$ and $F_{n}(\tilde{X}, \pi)$ are (path) connected and locally path connected for all compact polyhedra except when $X$ is homeomorphic to an interval or a circle. In the latter cases $F_{n}(X)$ and $F_{n}(\tilde{X}, \pi)$ are still locally path connected. In the general case, Theorem 2.3 of [16] describes a covering $F_{n}(\tilde{X}, \pi)$ of $D_{n}(X)$.

Theorem 2.1 (Xicoténcatl). Let $X$ be a compact polyhedron which is not homeomorphic to the circle or an interval, then there is a covering map

$$
p^{n}: F_{n}(\tilde{X}, \pi) \rightarrow D_{n}(X),
$$

with covering group the semidirect product $\pi_{1}(X)^{n} \rtimes \Sigma_{n}$, where $\pi_{1}(X)^{n}$ is the direct product of $n$ copies of the fundamental group and $p^{n}$ applies $p: \tilde{X} \rightarrow X$ to each element of an $n$-element configuration. The action of $\pi_{1}(X)^{n} \rtimes \Sigma_{n}$ on $F_{n}(\tilde{X}, \pi)$ is given by:

$$
\left(\alpha_{1}, \ldots, \alpha_{n} ; \sigma\right) \cdot\left(\tilde{x}_{1}, \ldots, \tilde{x}_{n}\right)=\left(\alpha_{1} \tilde{x}_{\sigma^{-1}(1)}, \ldots, \alpha_{n} \tilde{x}_{\sigma^{-1}(n)}\right) .
$$

The group operation and inverse for the semidirect product take the form:

$$
\begin{aligned}
\left(\alpha_{1}, \ldots, \alpha_{n} ; \sigma\right)\left(\beta_{1}, \ldots, \beta_{n} ; \rho\right) & =\left(\alpha_{1} \beta_{\sigma^{-1}(1)}, \ldots, \alpha_{n} \beta_{\sigma^{-1}(n)} ; \sigma \circ \rho\right) \\
\left(\alpha_{1}, \ldots, \alpha_{n} ; \sigma\right)^{-1} & =\left(\alpha_{\sigma(1)}^{-1}, \ldots, \alpha_{\sigma(n)}^{-1} ; \sigma^{-1}\right) .
\end{aligned}
$$

Remark 2.2. If $X$ is homeomorphic to an interval or a circle the space $F_{n}(\tilde{X}, \pi)$ is disconnected for all $n>1$. However the space $D_{n}(X)$ is connected (see Section [6). We still have that $\pi_{1}(X)^{n} \rtimes \Sigma_{n}$ acts properly discontinuously on $F_{n}(\tilde{X}, \pi)$ and that the orbit space $\left(\pi_{1}(X)^{n} \rtimes \Sigma_{n}\right) \backslash F_{n}(\tilde{X}, \pi)$ equals $D_{n}(X)$, but $\pi_{1}(X)^{n} \rtimes \Sigma_{n}$ is not the group of covering transformations. On the other hand we have the following facts

- if $C_{1}$ and $C_{2}$ are two connected components of $F_{n}(\tilde{X}, \pi)$, then there exists $\left(\alpha_{1}, \ldots, \alpha_{n} ; \sigma\right) \in \pi_{1}(X)^{n} \rtimes \Sigma_{n}$ such that

$$
\left(\alpha_{1}, \ldots, \alpha_{n} ; \sigma\right) C_{1}=C_{2} \text {. }
$$


- For any connected component $C$ of $F_{n}(\tilde{X}, \pi)$, it follows that the restriction map $p^{n}: C \rightarrow D_{n}(X)$ is a covering map, with covering group

$$
G=\left\{\left(\alpha_{1}, \ldots, \alpha_{n} ; \sigma\right) \in \pi_{1}(X)^{n} \rtimes \Sigma_{n} \mid\left(\alpha_{1}, \ldots, \alpha_{n} ; \sigma\right) C=C\right\} .
$$

Now, let $X$ be any compact polyhedron and let $f: X \rightarrow D_{n}(X)$ be an $n$-valued map and $\bar{f}: \tilde{X} \rightarrow F_{n}(\tilde{X}, \pi)$ a lifting of $f$. Since $F_{n}(\tilde{X}, \pi) \subseteq F_{n}(\tilde{X})$, we may write $\bar{f}$ in terms of the coordinate selfmaps of $\tilde{X}$ as $\bar{f}=\left(\bar{f}_{1}, \ldots, \bar{f}_{n}\right)$. Then we have the diagram

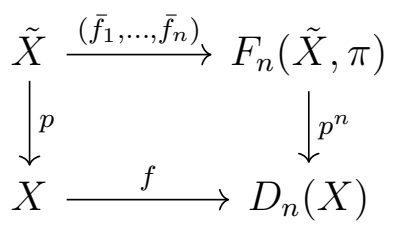

We call the maps $\bar{f}_{i}: \tilde{X} \rightarrow \tilde{X}$ the lift-factors of the map $f$. Note that another lifting $\bar{f}^{\prime}: \tilde{X} \rightarrow F_{n}(\tilde{X}, \pi)$ of $f$ gives rise to other liftfactors $\bar{f}_{1}^{\prime}, \ldots, \bar{f}_{n}^{\prime}$ of $f$. We define the set of lift-factors of $f$ as the set containing all lift-factors of all possible liftings of $f$.

Recall from the single-valued theory that, choosing a lifting $\tilde{f}^{*}: \tilde{X} \rightarrow$ $\tilde{X}$ to the universal covering space of a single-valued function $f: X \rightarrow$ $X$, the liftings are the $\alpha \tilde{f}^{*}$ where $\alpha$ is a covering transformation and therefore each lifting may be associated with an element of $\pi_{1}(X)$. Liftings $\alpha \tilde{f}^{*}$ and $\beta \tilde{f}^{*}$ are equivalent via $\mu \in \pi_{1}(X)$ if

$$
\alpha \tilde{f}^{*}=\mu \beta \tilde{f}^{*} \mu^{-1}
$$

In what follows, we will define a similar equivalence relation for $n$-valued maps on the set of lift-factors.

Lemma 2.3. Let $\bar{f}_{i}$ be a lift-factor of $f$ and $\gamma \in \pi_{1}(X)$. Then $\gamma \bar{f}_{i} \gamma^{-1}$ is also a lift-factor of $f$.

Proof. Let $\bar{f}: \tilde{X} \rightarrow F_{n}(\tilde{X}, \pi)$ be a lifting of $f$ with $i^{\text {th }}$ component $\bar{f}_{i}$. Consider the map

$$
(\gamma, \ldots, \gamma): F_{n}(\tilde{X}, \pi) \rightarrow F_{n}(\tilde{X}, \pi):\left(\tilde{x}_{1}, \ldots, \tilde{x}_{n}\right) \mapsto\left(\gamma \tilde{x}_{1}, \ldots, \gamma \tilde{x}_{n}\right)
$$

and the following commutative diagram (where $1_{X}$ is the identity on $X)$ :

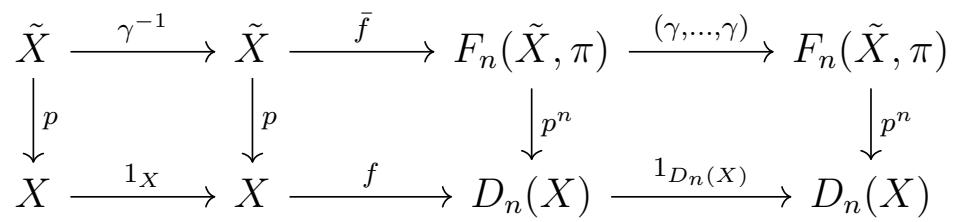


It follows that $(\gamma, \ldots, \gamma) \circ \bar{f} \circ \gamma^{-1}$ is a lifting of $f$. The $i^{\text {th }}$ component of this lifting is $\gamma \bar{f}_{i} \gamma^{-1}$, so this is a lift-factor of $f$.

Now we can define lift-factor equivalence on the set of lift-factors of $f$, denoted by $\sim_{l f}$, by

$$
\bar{f}_{i} \sim_{l f} \bar{f}_{j}^{\prime} \Longleftrightarrow \exists \gamma \in \pi_{1}(X): \bar{f}_{i}=\gamma \bar{f}_{j}^{\prime} \gamma^{-1}
$$

on the set of lift-factors of $f$.

The equivalence classes are called the lift-factor classes. We define the Reidemeister number $R(f)$ of $f: X \rightarrow D_{n}(X)$ to be the number of lift-factor classes. The Reidemeister number is either a natural number or $\infty$ and, since the lift-factors of a single-valued map are its liftings to the universal covering space, this is the classical concept if $f$ is single-valued.

Let $f: X \rightarrow D_{n}(X)$ be an $n$-valued map. We choose basepoints as follows. First select some $\tilde{x}^{*} \in \tilde{X}$ and let $x^{*}=p\left(\tilde{x}^{*}\right)$. Set $x^{(0)}=f\left(x^{*}\right) \in D_{n}(X)$ and choose $\tilde{x}^{(0)}=\left(\tilde{x}_{1}^{(0)}, \ldots, \tilde{x}_{n}^{(0)}\right) \in F(\tilde{X}, \pi)$ such that

$$
x^{(0)}=\left\{p\left(\tilde{x}_{1}^{(0)}\right), \ldots, p\left(\tilde{x}_{n}^{(0)}\right)\right\} .
$$

Let $\bar{f}^{*}: \tilde{X} \rightarrow F_{n}(\tilde{X}, \pi)$ be the lifting of $f$ such that $\bar{f}^{*}\left(\tilde{x}^{*}\right)=\tilde{x}^{(0)}$. Note that if $X$ is homeomorphic to the circle or an interval, there is still just one such lifting. The universal covering space $\tilde{X}$ is connected and thus $\bar{f}^{*}(\tilde{X})$ is also connected, so we can apply the usual covering space theory to the component $C$ of $F_{n}(\tilde{X}, \pi)$ which contains $\tilde{x}^{(0)}$.

The lifting $\bar{f}^{*}=\left(\bar{f}_{1}^{*}, \ldots, \bar{f}_{n}^{*}\right)$ is characterized by the property that it preserves basepoints, so we will call it the basic lifting of the $n$-valued map $f$. The lift-factors of $\bar{f}^{*}$ can be ordered such that $\bar{f}_{i}^{*}\left(\tilde{x}^{*}\right)=\tilde{x}_{i}^{(0)}$. For polyhedra $X$ not homeomorphic to the circle or an interval, by Theorem 2.1 and covering space theory all liftings of $f$ can be written in a unique way in the form

$$
\left(\alpha_{1}, \ldots, \alpha_{n} ; \eta\right) \bar{f}^{*}=\left(\alpha_{1} \bar{f}_{\eta^{-1}(1)}, \ldots, \alpha_{n} \bar{f}_{\eta^{-1}(n)}^{*}\right)
$$

for some $\left(\alpha_{1}, \ldots, \alpha_{n} ; \eta\right) \in \pi_{1}(X)^{n} \rtimes \Sigma_{n}$.

¿From Remark 2.2 it is not difficult to see that if $X$ is homeomorphic to the circle or an interval, each lifting of $f$ can be written uniquely in the same way.

It follows that every lift-factor of $f$ can be written as $\alpha \bar{f}_{i}^{*}$ for an $\alpha \in \pi_{1}(X)$ and $i \in\{1, \ldots, n\}$, so the set of lift-factors of $f$ is the set

$$
\left\{\alpha \bar{f}_{i}^{*} \mid \alpha \in \pi_{1}(X), i \in\{1, \ldots, n\}\right\} .
$$

In the next lemma we will show that if two lift-factors agree at any single point, then they must be the same. 
Lemma 2.4. Let $\bar{f}_{i}, \bar{f}_{j}^{\prime}: \tilde{X} \rightarrow \tilde{X}$ be lift-factors of $f$. If $\bar{f}_{i}(\tilde{x})=\bar{f}_{j}^{\prime}(\tilde{x})$ for any $\tilde{x} \in \tilde{X}$, then this holds for all $\tilde{x} \in \tilde{X}$ so $\bar{f}_{i}=\bar{f}_{j}^{\prime}$.

Proof. Let $\bar{f}^{*}=\left(\bar{f}_{1}^{*}, \ldots, \bar{f}_{n}^{*}\right)$ be a basic lifting of $f$. Then there exist $\alpha, \beta \in \pi_{1}(X)$ and $k, l \in\{1, \ldots, n\}$ such that $\bar{f}_{i}=\alpha \bar{f}_{k}^{*}$ and $\bar{f}_{j}^{\prime}=\beta \bar{f}_{l}^{*}$. For every $\tilde{x} \in \tilde{X}$ we have

$$
f(p(\tilde{x}))=p^{n}\left(\bar{f}^{*}(\tilde{x})\right)=\left\{p\left(\bar{f}_{1}^{*}(\tilde{x})\right), \ldots, p\left(\bar{f}_{n}^{*}(\tilde{x})\right)\right\} .
$$

Now assume that $\alpha \bar{f}_{k}^{*}(\tilde{x})=\beta \bar{f}_{l}^{*}(\tilde{x})$ for some $\tilde{x} \in \tilde{X}$, then

$$
p\left(\bar{f}_{k}^{*}(\tilde{x})\right)=p\left(\alpha \bar{f}_{k}^{*}(\tilde{x})\right)=p\left(\beta \bar{f}_{l}^{*}(\tilde{x})\right)=p\left(\bar{f}_{l}^{*}(\tilde{x})\right) .
$$

If $k \neq l$, then $f(p(\tilde{x}))$ would be a set with fewer than $n$ points of $X$. We conclude that $k=l$, so $\alpha \bar{f}_{k}^{*}(\tilde{x})=\beta \bar{f}_{k}^{*}(\tilde{x})$. Covering transformations are uniquely determined by the action on one point, so it follows that $\alpha=\beta$ and hence $\bar{f}_{i}=\bar{f}_{j}^{\prime}$.

In the lemma above we showed that for $\alpha, \beta \in \pi_{1}(X)$ and $i, j \in$ $\{1, \ldots, n\}$ :

$$
\alpha \bar{f}_{i}^{*}=\beta \bar{f}_{j}^{*} \Longleftrightarrow \alpha=\beta \text { and } i=j,
$$

so we can identify the set of lift-factors of $f$ with the set $\pi_{1}(X) \times$ $\{1, \ldots, n\}$.

The basic lifting $\bar{f}^{*}$ determines a function $\psi_{f}: \pi_{1}(X) \rightarrow \pi_{1}(X)^{n} \rtimes$ $\Sigma_{n}$ by the requirement that

$$
\forall \gamma \in \pi_{1}(X): \psi_{f}(\gamma) \circ \bar{f}^{*}=\bar{f}^{*} \circ \gamma .
$$

We write $\psi_{f}(\gamma)=\left(\phi_{1}(\gamma), \ldots, \phi_{n}(\gamma) ; \sigma_{\gamma}\right)$.

Lemma 2.5. The function $\psi_{f}: \pi_{1}(X) \rightarrow \pi_{1}(X)^{n} \rtimes \Sigma_{n}$ is a homomorphism.

Proof. Let $\alpha, \beta \in \pi_{1}(X)$ and $\tilde{x} \in \tilde{X}$. By the definition of $\psi_{f}$, we have on the one hand $\bar{f}^{*}(\alpha \beta \tilde{x})=\psi_{f}(\alpha \beta) \circ \bar{f}^{*}(\tilde{x})$ and on the other hand $\bar{f}^{*}(\alpha \beta \tilde{x})=\psi_{f}(\alpha) \circ \bar{f}^{*}(\beta \tilde{x})=\psi_{f}(\alpha) \circ \psi_{f}(\beta) \circ \bar{f}^{*}(\tilde{x})$. It follows that

$$
\psi_{f}(\alpha \beta)\left(\bar{f}_{1}^{*}(\tilde{x}), \ldots, \bar{f}_{n}^{*}(\tilde{x})\right)=\psi_{f}(\alpha) \psi_{f}(\beta)\left(\bar{f}_{1}^{*}(\tilde{x}), \ldots, \bar{f}_{n}^{*}(\tilde{x})\right) .
$$

Since any lifting of $f$ can be uniquely written in the form $\left(\alpha_{1}, \ldots, \alpha_{n} ; \eta\right) \bar{f}^{*}$, this implies that $\psi_{f}(\alpha \beta)=\psi_{f}(\alpha) \psi_{f}(\beta)$ which concludes the proof that $\psi_{f}$ is a homomorphism.

Note that the lemma implies that $\sigma: \pi_{1}(X) \rightarrow \Sigma_{n}: \gamma \mapsto \sigma_{\gamma}$ is also a homomorphism. 
Theorem 2.6. Let $f: X \rightarrow D_{n}(X)$ be an $n$-valued map with basic lifting $\bar{f}^{*}=\left(\bar{f}_{1}^{*}, \ldots, \bar{f}_{n}^{*}\right)$. Two lift-factors $\alpha \bar{f}_{i}^{*}$ and $\beta \bar{f}_{j}^{*}$ are equivalent if and only if there exists an element $\gamma \in \pi_{1}(X)$ such that

$$
\left\{\begin{array}{l}
\sigma_{\gamma}(j)=i \\
\alpha=\gamma \beta \phi_{j}\left(\gamma^{-1}\right)
\end{array}\right.
$$

Proof. Note that for all $\gamma \in \pi_{1}(X)$ :

$$
\begin{aligned}
\left(\bar{f}_{1}^{*}, \ldots, \bar{f}_{n}^{*}\right) \circ \gamma^{-1} & =\psi_{f}\left(\gamma^{-1}\right)\left(\bar{f}_{1}^{*}, \ldots, \bar{f}_{n}^{*}\right) \\
& =\left(\phi_{1}\left(\gamma^{-1}\right), \ldots, \phi_{n}\left(\gamma^{-1}\right) ; \sigma_{\gamma^{-1}}\right)\left(\bar{f}_{1}^{*}, \ldots, \bar{f}_{n}^{*}\right) \\
& =\left(\phi_{1}\left(\gamma^{-1}\right) \bar{f}_{\sigma_{\gamma}(1)}^{*}, \ldots, \phi_{n}\left(\gamma^{-1}\right) \bar{f}_{\sigma_{\gamma}(n)}^{*}\right) .
\end{aligned}
$$

The last equality holds because $\sigma$ is a homomorphism such that $\sigma_{\gamma^{-1}}^{-1}=\sigma_{\gamma}$. The $j^{t h}$ component of $\left(\bar{f}_{1}^{*}, \ldots, \bar{f}_{n}^{*}\right) \circ \gamma^{-1}$ is

$$
\bar{f}_{j}^{*} \circ \gamma^{-1}=\phi_{j}\left(\gamma^{-1}\right) \bar{f}_{\sigma_{\gamma}(j)}^{*} .
$$

This expression implies that for $\alpha, \beta \in \pi_{1}(X)$ and $i, j \in\{1, \ldots, n\}$ :

$$
\begin{aligned}
\alpha \bar{f}_{i}^{*} \sim_{l f} \beta \bar{f}_{j}^{*} & \Longleftrightarrow \exists \gamma \in \pi_{1}(X): \alpha \bar{f}_{i}^{*}=\gamma \beta \bar{f}_{j}^{*} \gamma^{-1} \\
& \Longleftrightarrow \exists \gamma \in \pi_{1}(X): \alpha \bar{f}_{i}^{*}=\gamma \beta \phi_{j}\left(\gamma^{-1}\right) \bar{f}_{\sigma_{\gamma}(j)}^{*} \\
& \Longleftrightarrow \exists \gamma \in \pi_{1}(X):\left\{\begin{array}{l}
\sigma_{\gamma}(j)=i \\
\alpha=\gamma \beta \phi_{j}\left(\gamma^{-1}\right),
\end{array}\right.
\end{aligned}
$$

where the last equivalence holds because $\alpha \bar{f}_{i}^{*}=\beta \bar{f}_{j}^{*} \Longleftrightarrow \alpha=$ $\beta$ and $i=j$.

Since we identify the set of lift-factors with the set $\pi_{1}(X) \times$ $\{1, \ldots, n\}$, the equivalence relation $\sim_{l f}$ gives rise to an equivalence relation on $\pi_{1}(X) \times\{1, \ldots, n\}$. We denote the equivalence classes of this relation by $[(\alpha, i)]$. Theorem 2.6 then implies that

$$
[(\alpha, i)]=[(\beta, j)] \Longleftrightarrow \exists \gamma \in \pi_{1}(X):\left\{\begin{array}{l}
\sigma_{\gamma}(j)=i \\
\alpha=\gamma \beta \phi_{j}\left(\gamma^{-1}\right) .
\end{array}\right.
$$

Theorem 2.7. If n-valued maps $f, g: X \rightarrow D_{n}(X)$ are homotopic, then $R(f)=R(g)$.

Proof. Let $f, g: X \rightarrow D_{n}(X)$ be homotopic $n$-valued maps, $\bar{f}^{*}$ the basic lifting of $f$ and $H: X \times I \rightarrow D_{n}(X)$ a homotopy such that $H(x, 0)=f(x)$ and $H(x, 1)=g(x)$ for all $x \in X$. Lifting $H$ to

$$
\bar{H}: \tilde{X} \times I \rightarrow F_{n}(\tilde{X}, \pi)
$$

with $\bar{H}(\tilde{x}, 0)=\bar{f}^{*}(\tilde{x})$ defines the basic lifting $\bar{g}^{*}$ of $g$ by setting $\bar{H}(\tilde{x}, 1)=\bar{g}^{*}(\tilde{x})$. If $X$ is homeomorphic to the circle or an interval 
we can use the usual covering space theory by restricting $F_{n}(\tilde{X}, \pi)$ to the connected component $C$ of $F_{n}(\tilde{X}, \pi)$ containing $\bar{f}^{*}(\tilde{x})$. Considering the basic liftings $\bar{f}^{*}$ of $f$ and $\bar{g}^{*}$ of $g$, we will show that $\psi_{f}=\psi_{g}$. Let $\gamma \in \pi_{1}(X)$ and define the map

$$
\gamma \times 1_{I}: \tilde{X} \times I \rightarrow \tilde{X} \times I:(\tilde{x}, t) \mapsto(\gamma \tilde{x}, t) .
$$

The map $\bar{H} \circ\left(\gamma \times 1_{I}\right): \tilde{X} \times I \rightarrow F_{n}(\tilde{X}, \pi)$ is a lifting of $H$ with

$$
\begin{array}{r}
\left(\bar{H} \circ\left(\gamma \times 1_{I}\right)\right)(\tilde{x}, 0)=\bar{H}(\gamma \tilde{x}, 0)=\bar{f}^{*}(\gamma \tilde{x})=\psi_{f}(\gamma) \bar{f}^{*}(\tilde{x}), \\
\left(\bar{H} \circ\left(\gamma \times 1_{I}\right)\right)(\tilde{x}, 1)=\bar{H}(\gamma \tilde{x}, 1)=\bar{g}^{*}(\gamma \tilde{x})=\psi_{g}(\gamma) \bar{g}^{*}(\tilde{x})
\end{array}
$$

for all $\tilde{x} \in \tilde{X}$. The map $\psi_{f}(\gamma) \circ \bar{H}$ is also a lifting of $H$, with

$$
\begin{aligned}
\left(\psi_{f}(\gamma) \circ \bar{H}\right)(\tilde{x}, 0) & =\psi_{f}(\gamma) \bar{f}^{*}(\tilde{x}), \\
\left(\psi_{f}(\gamma) \circ \bar{H}\right)(\tilde{x}, 1) & =\psi_{f}(\gamma) \bar{g}^{*}(\tilde{x}),
\end{aligned}
$$

for all $\tilde{x} \in \tilde{X}$. By the uniqueness of lifting property (and the fact that all maps have there image in the same connected component $C$ in the case that $X$ is homeomorphic to a circle or an interval), it follows that $\psi_{g}(\gamma) \bar{g}^{*}(\tilde{x})=\psi_{f}(\gamma) \bar{g}^{*}(\tilde{x})$ for all $\tilde{x} \in \tilde{X}$ and, applying uniqueness of liftings again, this shows that $\psi_{f}=\psi_{g}$. Theorem 2.6 then implies that $R(f)=R(g)$.

Theorem 2.8. Let $f: X \rightarrow D_{n}(X)$ be an $n$-valued map, then

$$
\operatorname{Fix}(f)=\bigcup_{\bar{f}_{i}} p \operatorname{Fix}\left(\bar{f}_{i}\right)
$$

where the union is taken over the set of lift-factors of $f$ and $\operatorname{Fix}(f)=$ $\{x \in X \mid x \in f(x)\}$.

Proof. Let $\tilde{x} \in \tilde{X}$ such that $\tilde{x}=\bar{f}_{i}(\tilde{x})$. Then for $x=p(\tilde{x})$, we have

$$
x=p\left(\bar{f}_{i}(\tilde{x})\right) \in f p(\tilde{x})=f(x)
$$

and we have proved that

$$
\bigcup_{\bar{f}_{i}} p \operatorname{Fix}\left(\bar{f}_{i}\right) \subseteq \operatorname{Fix}(f) .
$$

Now suppose $x \in \operatorname{Fix}(f)$. Choose a lifting $\bar{f}=\left(\bar{f}_{1}, \ldots, \bar{f}_{n}\right): \tilde{X} \rightarrow$ $F_{n}(\tilde{X}, \pi)$ of $f$ and let

$$
p^{n} \bar{f}=\left\{p \bar{f}_{1}, \ldots, p \bar{f}_{n}\right\}: \tilde{X} \rightarrow D_{n}(X) .
$$

Since $x$ is a fixed point, there exists $i \in\{1, \ldots, n\}$ and $\tilde{x} \in p^{-1}(x)$ such that $p \bar{f}_{i}(\tilde{x})=x$ so $\bar{f}_{i}(\tilde{x}) \in p^{-1}(x)$ and thus there exists $\gamma \in$ 
$\pi_{1}(X)$ such that $\gamma \bar{f}_{i}(\tilde{x})=\tilde{x}$. We have proved that $x \in p \operatorname{Fix}\left(\gamma \bar{f}_{i}\right)$ and since $\gamma \bar{f}_{i}$ is also a lift-factor of $f$, we have

$$
\operatorname{Fix}(f) \subseteq \bigcup_{\bar{f}_{i}} p \operatorname{Fix}\left(\bar{f}_{i}\right) .
$$

In the next theorem we will see that an equivalence class of liftfactors determines a subset of $\operatorname{Fix}(f)$.

Theorem 2.9. Let $f: X \rightarrow D_{n}(X)$ be an $n$-valued map and $\bar{f}_{i}, \bar{f}_{j}^{\prime}$ two lift-factors of $f$.

(a) If $\bar{f}_{i} \sim_{l f} \bar{f}_{j}^{\prime}$, then $p \operatorname{Fix}\left(\bar{f}_{i}\right)=p \operatorname{Fix}\left(\bar{f}_{j}^{\prime}\right)$.

(b) If $p \operatorname{Fix}\left(\bar{f}_{i}\right) \cap p \operatorname{Fix}\left(\bar{f}_{j}^{\prime}\right) \neq \emptyset$ then $\bar{f}_{i} \sim_{l f} \bar{f}_{j}^{\prime}$.

Proof. For $(a)$, assume that $\bar{f}_{i}=\gamma \bar{f}_{j}^{\prime} \gamma^{-1}$ with $\gamma \in \pi_{1}(X)$. We will show that $p \operatorname{Fix}\left(\bar{f}_{i}\right)=p \operatorname{Fix}\left(\bar{f}_{j}^{\prime}\right)$. Let $x_{0} \in p \operatorname{Fix}\left(\bar{f}_{i}\right)$. Then there exists $\tilde{x}_{0} \in p^{-1}\left(x_{0}\right) \cap \operatorname{Fix}\left(\bar{f}_{i}\right)$ so

$$
\tilde{x}_{0}=\bar{f}_{i}\left(\tilde{x}_{0}\right)=\gamma \bar{f}_{j}^{\prime}\left(\gamma^{-1} \tilde{x}_{0}\right) .
$$

Therefore $\gamma^{-1} \tilde{x}_{0}=\bar{f}_{j}^{\prime}\left(\gamma^{-1} \tilde{x}_{0}\right)$, that is $\gamma^{-1} \tilde{x}_{0} \in \operatorname{Fix}\left(\bar{f}_{j}^{\prime}\right)$, and thus $x_{0}=$ $p\left(\gamma^{-1} \tilde{x}_{0}\right) \in p \operatorname{Fix}\left(\bar{f}_{j}^{\prime}\right)$. We have proved that $p \operatorname{Fix}\left(\bar{f}_{i}\right) \subseteq p \operatorname{Fix}\left(\bar{f}_{j}^{\prime}\right)$. A symmetric argument establishes that $p \operatorname{Fix}\left(\bar{f}_{i}\right)=p \operatorname{Fix}\left(\bar{f}_{j}^{\prime}\right)$.

Now, for (b), we assume that $p \operatorname{Fix}\left(\bar{f}_{i}\right) \cap p \operatorname{Fix}\left(\bar{f}_{j}^{\prime}\right)$ is nonempty and we will find an element $\gamma \in \pi_{1}(X)$ such that $\bar{f}_{i}=\gamma \bar{f}_{j}^{\prime} \gamma^{-1}$. Let $x_{0} \in p \operatorname{Fix}\left(\bar{f}_{i}\right) \cap p \operatorname{Fix}\left(\bar{f}_{j}^{\prime}\right)$ and choose $\tilde{x}_{0} \in p^{-1}\left(x_{0}\right) \cap \operatorname{Fix}\left(\bar{f}_{i}\right)$. There exists $\mu \in \pi_{1}(X)$ such that $\mu \tilde{x}_{0} \in \operatorname{Fix}\left(\bar{f}_{j}^{\prime}\right)$, so $\bar{f}_{j}^{\prime}\left(\mu \tilde{x}_{0}\right)=\mu \tilde{x}_{0}$ and thus

$$
\mu^{-1} \bar{f}_{j}^{\prime}\left(\mu \tilde{x}_{0}\right)=\tilde{x}_{0}=\bar{f}_{i}\left(\tilde{x}_{0}\right) .
$$

Since the lift-factors $\mu^{-1} \bar{f}_{j}^{\prime} \mu$ and $\bar{f}_{i}$ agree at a point, they are the same by Lemma 2.4, so $\mu^{-1} \bar{f}_{j}^{\prime} \mu=\bar{f}_{i}$. Choosing $\gamma=\mu^{-1}$, this completes the proof.

For a map $f: X \rightarrow D_{n}(X)$, we can now define the fixed point classes of $f$. To any equivalence class $\left[\bar{f}_{i}\right]$ of a lift-factor, we associate a fixed point class which is the subset of $\operatorname{Fix}(f)$ given by

$$
p \operatorname{Fix}\left(\bar{f}_{i}\right)=\left\{p(\tilde{x}) \mid \bar{f}_{i}(\tilde{x})=\tilde{x}\right\} \subseteq \operatorname{Fix}(f) .
$$

The theorem above shows that this subset does not depend on the chosen representative of the equivalence class $\left[\bar{f}_{i}\right]$.

We note that $\operatorname{Fix}\left(\bar{f}_{i}\right)$ may be empty and therefore a fixed point class $p \operatorname{Fix}\left(\bar{f}_{i}\right)$ may be the empty set. Just as in the single-valued 
case, two empty fixed point classes that are determined by different lift-factor classes will be regarded as being different fixed point classes.

Thus two nonempty fixed point classes $p \operatorname{Fix}\left(\bar{f}_{i}\right)$ and $p \operatorname{Fix}\left(\bar{f}_{j}^{\prime}\right)$ are equal if and only if $\bar{f}_{i} \sim_{l f} \bar{f}_{j}^{\prime}$ and are disjoint when $\bar{f}_{i} \nsim_{l f} \bar{f}_{j}^{\prime}$. So $R(f)$, the number of lift-factor classes, is also the number of fixed point classes.

\section{The Construction of Dugardein}

We continue to denote the universal covering space of $X$ by $p: \tilde{X} \rightarrow$ $X$. Given an $n$-valued map $f: X \rightarrow D_{n}(X)$, Gert-Jan Dugardein defined a map $\hat{f}: \tilde{X} \rightarrow F_{n}(\tilde{X}, \pi)$ as follows 1 The map $f p: \tilde{X} \rightarrow$ $D_{n}(X)$ lifts to $F_{n}(X)$ and therefore it splits as $\left(f_{1}, \ldots, f_{n}\right): \tilde{X} \rightarrow$ $F_{n}(X)$ where $f_{i}: \tilde{X} \rightarrow X$ for each $i$. The ordering can be chosen so that each $f_{i}$ lifts to $\tilde{f}_{i}: \tilde{X} \rightarrow \tilde{X}$ such that $\tilde{f}_{i}\left(\tilde{x}^{*}\right)=\tilde{x}_{i}^{(0)}$, the basepoints of Section 2. Therefore, $\left(f_{1}, \ldots, f_{n}\right)$ lifts to $\hat{f}=$ $\left(\tilde{f}_{1}, \ldots, \tilde{f}_{n}\right): \tilde{X} \rightarrow F_{n}(\tilde{X})$ such that $\hat{f}\left(\tilde{x}^{*}\right)=\tilde{x}^{(0)}$. Since $p^{n}\left(\tilde{f}_{1}, \ldots, \tilde{f}_{n}\right)(\tilde{x}) \in$ $D_{n}(X)$ for all $\tilde{x} \in \tilde{X}$ and hence $p \tilde{f}_{i}(\tilde{x}) \neq p \tilde{f}_{j}(\tilde{x})$ for $i \neq j$, we may consider $\hat{f}$ as a map $\hat{f}: \tilde{X} \rightarrow F_{n}(\tilde{X}, \pi)$.

It is clear from the definition that $\hat{f}: \tilde{X} \rightarrow F_{n}(\tilde{X}, \pi)$ is a lifting of $f: X \rightarrow D_{n}(X)$. The basic lifting $\bar{f}^{*}$ is also such a lifting and $\hat{f}\left(\tilde{x}^{*}\right)=\bar{f}^{*}\left(\tilde{x}^{*}\right)=\tilde{x}^{(0)}$, so they are the same map. Moreover, since $\left(\tilde{f}_{1}, \ldots, \tilde{f}_{n}\right)$ and $\left(\bar{f}_{1}^{*}, \ldots, \bar{f}_{n}^{*}\right)$ are two splittings of $\bar{f}=\hat{f}$ that correspond at the basepoints, then $\tilde{f}_{i}=\bar{f}_{i}^{*}$ for $i=1, \ldots, n$. Consequently, the results of this section, that concern the subsets $p \operatorname{Fix}\left(\alpha_{i} \tilde{f}_{i}\right)$ of Fix $(f)$, apply as well to the fixed point classes that were defined in Section 2 as the sets $p \operatorname{Fix}\left(\alpha_{i} \bar{f}_{i}^{*}\right)$.

For a map $f: X \rightarrow D_{n}(X)$, employing the definition of Schirmer in [14] we will say that $x_{0}, x_{1} \in \operatorname{Fix}(f)$ are S-equivalent if there is a map $c: I=[0,1] \rightarrow X$ from $x_{0}$ to $x_{1}$ such that, for the splitting $f c=\left\{c_{1}, \ldots, c_{n}\right\}: I \rightarrow D_{n}(X)$, some $c_{k}$ is a path from $x_{0}$ to $x_{1}$ and $c_{k}$ is homotopic to $c$ relative to the endpoints.

Theorem 3.1 (Dugardein). Fixed points $x_{0}, x_{1}$ of $f: X \rightarrow D_{n}(X)$ are S-equivalent if and only if there exists $i \in\{1, \ldots, n\}$ and $\alpha_{i} \in$ $\pi_{1}(X)$ such that $x_{0}, x_{1} \in p \operatorname{Fix}\left(\alpha_{i} \tilde{f}_{i}\right)$.

\footnotetext{
${ }^{1}$ The construction and its properties were presented at the conference "Nielsen Theory and Related Topics" held in Rio Claro, Brazil in July, 2016. Dugardein has made the slides of his talk available to the authors and given them permission to include this material in the present paper.
} 
Proof. Suppose $x_{0}, x_{1} \in p \operatorname{Fix}\left(\alpha_{i} \tilde{f}_{i}\right)$ so there exist $\tilde{x}_{0} \in p^{-1}\left(x_{0}\right), \tilde{x}_{1} \in$ $p^{-1}\left(x_{1}\right)$ in $\tilde{X}$ such that $\tilde{x}_{0}=\alpha_{i} \tilde{f}_{i}\left(\tilde{x}_{0}\right)$ and $\tilde{x}_{1}=\alpha_{i} \tilde{f}_{i}\left(\tilde{x}_{1}\right)$. Let $\tilde{c}: I \rightarrow$ $\tilde{X}$ be a path from $\tilde{x}_{0}$ to $\tilde{x}_{1}$, then $\tilde{c}$ is homotopic to $\alpha_{i} \tilde{f}_{i} \tilde{c}$ relative to the endpoints by a homotopy $\tilde{H}: I \times I \rightarrow \tilde{X}$. Let $p(\tilde{c})=c$, then $f c: I \rightarrow D_{n}$ splits as $f c=\left\{c_{1}, \ldots, c_{n}\right\}$. We have a commutative diagram:

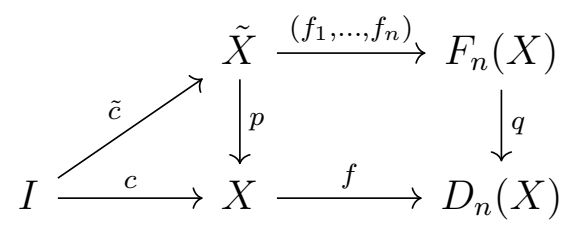

Now $p \tilde{H}: I \times I \rightarrow X$ is a homotopy between $c$ and $p \alpha_{i} \tilde{f}_{i} \tilde{c}$. On the other hand,

$$
p \alpha_{i} \tilde{f}_{i} \tilde{c}=p \tilde{f}_{i} \tilde{c}=f_{i} \tilde{c} \in q\left(f_{1}, \ldots, f_{n}\right) \tilde{c}=f c
$$

so $p \alpha_{i} \tilde{f}_{i} \tilde{c}=c_{k}$ for some $k \in\{1, \ldots, n\}$ and therefore $x_{0}$ and $x_{1}$ are S-equivalent.

If fixed points $x_{0}, x_{1}$ of $f: X \rightarrow D_{n}(X)$ are S-equivalent, then there is a map $c: I \rightarrow X$ from $x_{0}$ to $x_{1}$ such that, for the splitting $f c=\left\{c_{1}, \ldots, c_{n}\right\}: I \rightarrow D_{n}(X)$, some $c_{k}$ is a path from $x_{0}$ to $x_{1}$ and $c_{k}$ is homotopic to $c$ relative to the endpoints. Let $H: I \times I \rightarrow X$ be a homotopy from $c_{k}$ to $c$ relative to the endpoints, that is, $H(0, t)=$ $x_{0}, H(1, t)=x_{1}$ for all $t \in I$ and $H(s, 0)=c_{k}(s), H(s, 1)=c(s)$ for all $s \in I$. Choose some $\tilde{x}_{0} \in p^{-1}\left(x_{0}\right)$. Since $\left(\tilde{f}_{1}, \ldots, \tilde{f}_{n}\right)$ is a lifting of $f$ and $x_{0}$ is a fixed point of $f$, then there exists $i \in\{1, \ldots, n\}$ such that $\tilde{f}_{i}\left(\tilde{x}_{0}\right) \in p^{-1}\left(x_{0}\right)$ and therefore $\alpha_{i} \in \pi_{1}(X)$ such that $\alpha_{i} \tilde{f}_{i}\left(\tilde{x}_{0}\right)=\tilde{x}_{0}$. Let $\tilde{H}: I \times I \rightarrow \tilde{X}$ be the lifting of $H$ to $\tilde{x}_{0}$ such that $\tilde{H}(0, t)=\tilde{x}_{0}$ for all $t \in I$. Define $\tilde{x}_{1}=\tilde{H}(1,0)$ and thus $\tilde{H}(1, t)=\tilde{x}_{1}$ for all $t \in I$. Note that $p\left(\tilde{x}_{1}\right)=x_{1}$.

The path $\tilde{c}$, which is defined as the restriction of $\tilde{H}$ to $I \times\{1\}$ is a lifting of the path $c$ starting at $\tilde{x}_{0}$.

We claim that the path $\tilde{c}^{\prime}$, defined as $\tilde{H}$ restricted to $I \times\{0\}$, is

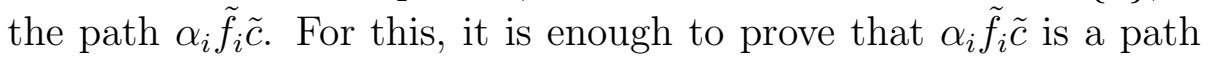
starting at $\tilde{x}_{0}$ and is a lifting of the path $c_{k}$. The starting point of $\alpha_{i} \tilde{f}_{i} \tilde{c}$ is $\alpha_{i} \tilde{f}_{i} \tilde{c}(0)=\alpha_{i} \tilde{f}_{i}\left(\tilde{x}_{0}\right)=\tilde{x}_{0}$. As above we have that

$$
p \alpha_{i} \tilde{f}_{i} \tilde{c}=p \tilde{f}_{i} \tilde{c}=f_{i} \tilde{c} \in q\left(f_{1}, \ldots, f_{n}\right) \tilde{c}=f c .
$$

And so $p \alpha_{i} \tilde{f}_{i} \tilde{c}$ is one of the paths $c_{j}$. But we know that $p \alpha_{i} \tilde{f}_{i} \tilde{c}$ starts at $x_{0}$. Since each of the paths $c_{j}$ starts at a different point, we must have that $p \alpha_{i} \tilde{f}_{i} \tilde{c}$ is equal to the unique path which also starts at $x_{0}$, which is $c_{k}$, and this proves our claim. 
Since $\tilde{H}$ is a path homotopy between $\tilde{c}$ and $\tilde{c}^{\prime}=\alpha_{i} \tilde{f}_{i} \tilde{c}$, it follows that $\tilde{c}(1)=\tilde{c}^{\prime}(1)$, so

$$
\tilde{x}_{1}=\tilde{c}(1)=\tilde{c}^{\prime}(1)=\alpha_{i} \tilde{f}_{i}\left(\tilde{x}_{1}\right)
$$

which shows that $\tilde{x}_{1} \in \operatorname{Fix}\left(\alpha_{i} \tilde{f}_{i}\right)$. So we have proved that $x_{0}, x_{1} \in$ $p \operatorname{Fix}\left(\alpha_{i} \tilde{f}_{i}\right)$.

Thus by Theorem 3.1 the fixed point classes $p \operatorname{Fix}\left(\alpha_{i} \bar{f}_{i}^{*}\right)$ defined in Section 2 are the same subsets of $\operatorname{Fix}(f)$ as those of Schirmer in [14]. Schirmer in [14] defined the Nielsen number $N(f)$ of an $n$-valued map to be the number of fixed point classes of non-zero index. By Theorem 2.9 we can conclude that

Proposition 3.2. For any $n$-valued map $f: X \rightarrow D_{n}(X)$, we have $N(f) \leq R(f)$.

\section{Computation of the Reidemeister number}

In the previous section, we proved that the Reidemeister number of an $n$-valued map is an upper bound for the Nielsen number. In this section, we will present a method for computing the Reidemeister number and we will demonstrate this method by means of the following example of a 3 -valued map on the torus $T^{2}=S^{1} \times S^{1}$. (We will view $S^{1}$ as being the set of complex numbers of modulus 1 ).

Consider the maps $\bar{f}_{i}^{*}: \mathbb{R}^{2} \rightarrow \mathbb{R}^{2}(i=1,2,3)$ defined by

$$
\begin{aligned}
& \bar{f}_{1}^{*}(t, s)=\left(\frac{t}{2},-s\right), \\
& \bar{f}_{2}^{*}(t, s)=\left(\frac{t+1}{2},-s\right), \\
& \bar{f}_{3}^{*}(t, s)=\left(-t,-s+\frac{1}{2}\right) .
\end{aligned}
$$

These maps induce a 3-valued map on the torus:

$$
f: T^{2} \multimap T^{2}: p(t, s) \mapsto\left\{p\left(\frac{t}{2},-s\right), p\left(\frac{t+1}{2},-s\right), p\left(-t,-s+\frac{1}{2}\right)\right\},
$$

where $p: \mathbb{R}^{2} \rightarrow T^{2}:(t, s) \mapsto\left(e^{2 \pi i t}, e^{2 \pi i s}\right)$ is the universal covering space. If we choose basepoints $\tilde{x}^{*}=(0,0), x^{*}=p(0,0), x^{(0)}=$ $f\left(x^{*}\right)=\left\{p(0,0), p\left(\frac{1}{2}, 0\right), p\left(0, \frac{1}{2}\right)\right\}$ and $\tilde{x}^{(0)}=\left((0,0),\left(\frac{1}{2}, 0\right),\left(0, \frac{1}{2}\right)\right)$, then $\bar{f}^{*}=\left(\bar{f}_{1}^{*}, \bar{f}_{2}^{*}, \bar{f}_{3}^{*}\right): \mathbb{R}^{2} \rightarrow F_{3}\left(\mathbb{R}^{2}, \pi_{1}\left(T^{2}\right)\right)$ is the basic lifting of $f$. We start by computing the fixed points of $f$. A point $p(t, s) \in T^{2}$ is a 
fixed point of $f$ if and only if $p(t, s)=p\left(\frac{t}{2},-s\right)$ or $p(t, s)=p\left(\frac{t+1}{2},-s\right)$ or $p(t, s)=p\left(-t,-s+\frac{1}{2}\right)$.

We consider the three cases separately. For the first case, we have

$$
\begin{aligned}
p(t, s)=p\left(\frac{t}{2},-s\right) & \Longleftrightarrow \exists k, m \in \mathbb{Z}: t=\frac{t}{2}+k \text { and } s=-s+m \\
& \Longleftrightarrow \frac{t}{2} \in \mathbb{Z} \text { and } 2 s \in \mathbb{Z}
\end{aligned}
$$

The fixed points we find in this way are $p(0,0)$ and $p\left(0, \frac{1}{2}\right)$.

Analogously, the requirement that $p(t, s)=p\left(\frac{t+1}{2},-s\right)$ leads to the same fixed points $p(0,0)(=p(1,0))$ and $p\left(0, \frac{1}{2}\right)\left(=p\left(1, \frac{1}{2}\right)\right)$.

Finally, requiring that $p(t, s)=p\left(-t,-s+\frac{1}{2}\right)$ gives rise to four fixed points $p\left(0, \frac{1}{4}\right), p\left(0, \frac{3}{4}\right), p\left(\frac{1}{2}, \frac{1}{4}\right)$ and $p\left(\frac{1}{2}, \frac{3}{4}\right)$.

So $f$ has six fixed points, of which two are related to both $\bar{f}_{1}^{*}$ and $\bar{f}_{2}^{*}$ and the other four are connected with $\bar{f}_{3}^{*}$. It is important to note that $\bar{f}_{1}^{*}$ and $\bar{f}_{2}^{*}$ give rise to the same fixed points. $\Sigma_{n}$ :

Recall the definition of the homomorphism $\psi_{f}: \pi_{1}(X) \rightarrow \pi_{1}(X)^{n} \rtimes$

$$
\forall \gamma \in \pi_{1}(X): \psi_{f}(\gamma) \circ \bar{f}^{*}=\bar{f}^{*} \circ \gamma
$$

We will compute $\psi_{f}$ for this 3 -valued map $f$. Let $\gamma \in \mathbb{Z}^{2}$, so $\gamma=$ $\left(z_{1}, z_{2}\right)$ which acts on $(x, y) \in \mathbb{R}^{2}$ by translation. Then

$$
\begin{aligned}
& \left(\bar{f}^{*} \circ \gamma\right)(t, s)=\bar{f}^{*}\left(t+z_{1}, s+z_{2}\right) \\
& =\left(\left(\frac{t+z_{1}}{2},-s-z_{2}\right),\left(\frac{t+z_{1}+1}{2},-s-z_{2}\right),\left(-t-z_{1},-s-z_{2}+\frac{1}{2}\right)\right) .
\end{aligned}
$$

When $z_{1}$ is even, we find that this equals

$$
\left(\left(\frac{z_{1}}{2},-z_{2}\right),\left(\frac{z_{1}}{2},-z_{2}\right),\left(-z_{1},-z_{2}\right) ; I\right) \bar{f}^{*}(t, s),
$$

with $I$ the identity permutation of $\{1,2,3\}$, while if $z_{1}$ is odd, this equals

$$
\left(\left(\frac{z_{1}-1}{2},-z_{2}\right),\left(\frac{z_{1}+1}{2},-z_{2}\right),\left(-z_{1},-z_{2}\right) ; \nu\right) \bar{f}^{*}(t, s),
$$

with $\nu(1)=2, \nu(2)=1$ and $\nu(3)=3$. This determines $\psi_{f}(\gamma)=$ $\left(\phi_{1}(\gamma), \phi_{2}(\gamma), \phi_{3}(\gamma) ; \sigma_{\gamma}\right)$ for all $\gamma=\left(z_{1}, z_{2}\right) \in \mathbb{Z}^{2}$ :

\begin{tabular}{r|c|c} 
& $z_{1}$ even & $z_{1}$ odd \\
\hline$\phi_{1}\left(z_{1}, z_{2}\right)$ & $\left(\frac{z_{1}}{2},-z_{2}\right)$ & $\left(\frac{z_{1}-1}{2},-z_{2}\right)$ \\
$\phi_{2}\left(z_{1}, z_{2}\right)$ & $\left(\frac{z_{1}}{2},-z_{2}\right)$ & $\left(\frac{z_{1}+1}{2},-z_{2}\right)$ \\
$\phi_{3}\left(z_{1}, z_{2}\right)$ & $\left(-z_{1},-z_{2}\right)$ & $\left(-z_{1},-z_{2}\right)$ \\
$\sigma_{\left(z_{1}, z_{2}\right)}$ & $I$ & $\nu=(12)(3)$
\end{tabular}


We now turn back to the general situation. Each time we consider a map $f: X \rightarrow D_{n}(X)$, we will assume that a basic lifting $\bar{f}^{*}$ as in Section 2 is fixed and we will also consider the induced homomorphism $\psi_{f}: \pi_{1}(X) \rightarrow \pi_{1}(X)^{n} \rtimes \Sigma_{n}$ with $\psi_{f}(\gamma)=$ $\left(\phi_{1}(\gamma), \ldots, \phi_{n}(\gamma) ; \sigma_{\gamma}\right)$.

Lemma 4.1. Let $f: X \rightarrow D_{n}(X)$ be an $n$-valued map with basic lifting $\bar{f}^{*}$. Let $i, j \in\{1, \ldots, n\}$ and suppose there exists $\gamma \in \pi_{1}(X)$ such that $\sigma_{\gamma}(j)=i$. Then for every $\alpha \in \pi_{1}(X)$ there exists $\beta \in$ $\pi_{1}(X)$ such that $\alpha \bar{f}_{i}^{*} \sim_{l f} \beta \bar{f}_{j}^{*}$.

Proof. If $\gamma \in \pi_{1}(X)$ such that $\sigma_{\gamma}(j)=i$, then $j=\sigma_{\gamma}^{-1}(i)$. By the definition of $\psi_{f}$, we have $\bar{f}^{*} \circ \gamma=\psi_{f}(\gamma) \bar{f}^{*}$. If we write this in components, we have

$$
\left(\bar{f}_{1}^{*} \gamma, \ldots, \bar{f}_{n}^{*} \gamma\right)=\left(\phi_{1}(\gamma) \bar{f}_{\sigma_{\gamma}^{-1}(1)}^{*}, \ldots, \phi_{n}(\gamma) \bar{f}_{\sigma_{\gamma}^{-1}(n)}\right) .
$$

The $i^{\text {th }}$ component is $\bar{f}_{i}^{*} \gamma=\phi_{i}(\gamma) \bar{f}_{\sigma_{\gamma}^{-1}(i)}=\phi_{i}(\gamma) \bar{f}_{j}^{*}$. It follows that

$$
\alpha \bar{f}_{i}^{*}=\alpha \phi_{i}(\gamma) \bar{f}_{j}^{*} \gamma^{-1}=\gamma \gamma^{-1} \alpha \phi_{i}(\gamma) \bar{f}_{j}^{*} \gamma^{-1}
$$

Choosing $\beta=\gamma^{-1} \alpha \phi_{i}(\gamma)$, we have proved that $\alpha \bar{f}_{i}^{*} \sim_{l f} \beta \bar{f}_{j}^{*}$.

For $i, j \in\{1, \ldots, n\}$, we will write $i \sim j$ when there is some $\gamma \in \pi_{1}(X)$ with $\sigma_{\gamma}(j)=i$. This defines an equivalence relation because $\sigma$ is a homomorphism. The relation $\sim$ divides the basic lifting $\bar{f}^{*}=\left(\bar{f}_{1}^{*}, \ldots, \bar{f}_{n}^{*}\right)$ into equivalence classes where $\bar{f}_{i}^{*}$ and $\bar{f}_{j}^{*}$ are in the same class when $i \sim j$. We will refer to these classes as the $\sigma$-classes of the basic lifting.

Now we compute the $\sigma$-classes for the 3 -valued map $f$ of the example. For $\left(z_{1}, z_{2}\right) \in \mathbb{Z}^{2}$, we showed before that $\sigma_{\left(z_{1}, z_{2}\right)}=I$ if $z_{1}$ is even and $\sigma_{\left(z_{1}, z_{2}\right)}=(12)(3)$ if $z_{1}$ is odd. Consequently the basic lifting $\bar{f}^{*}$ has two $\sigma$-classes : $\left\{\bar{f}_{1}^{*}, \bar{f}_{2}^{*}\right\}$ and $\left\{\bar{f}_{3}^{*}\right\}$.

In general, let $r$ be the number of $\sigma$-classes and take for every class a representative $\bar{f}_{i_{k}}^{*}$ so that we have the set of representatives $\left\{\bar{f}_{i_{1}}^{*}, \bar{f}_{i_{2}}^{*}, \ldots, \bar{f}_{i_{r}}^{*}\right\}$. By Lemma 4.1 and Theorem 2.6,

$$
R(f)=R_{1}(f)+\cdots+R_{r}(f),
$$

with $R_{k}(f)$ the number of equivalence classes of the restriction of the relation $\sim_{l f}$ to the set $\left\{\alpha \bar{f}_{i_{k}}^{*} \mid \alpha \in \pi_{1}(X)\right\}$. For $i \in\{1, \ldots, n\}$, define the following finite index subgroup of $\pi_{1}(X)$ :

$$
S_{i}=\left\{\gamma \in \pi_{1}(X) \mid \sigma_{\gamma}(i)=i\right\} .
$$


For the computation of $R_{k}(f)$, we restrict $\sim_{l f}$ to the set $\left\{\alpha \bar{f}_{i_{k}}^{*} \mid \alpha \in\right.$ $\left.\pi_{1}(X)\right\}$ and we find that

$$
\begin{aligned}
\alpha \bar{f}_{i_{k}}^{*} \sim_{l f} \beta \bar{f}_{i_{k}}^{*} & \Longleftrightarrow \exists \gamma \in \pi_{1}(X):\left\{\begin{array}{l}
\sigma_{\gamma}\left(i_{k}\right)=i_{k} \\
\alpha=\gamma \beta \phi_{i_{k}}\left(\gamma^{-1}\right)
\end{array}\right. \\
& \Longleftrightarrow \exists \gamma \in S_{i_{k}}: \alpha=\gamma \beta \phi_{i_{k}}\left(\gamma^{-1}\right) .
\end{aligned}
$$

Note that, in general, the map $\phi_{i}: \pi_{1}(X) \rightarrow \pi_{1}(X)$ is not a homomorphism, but that if we restrict the domain to $S_{i}$, then $\phi_{i}$ : $S_{i} \rightarrow \pi_{1}(X)$ will be a homomorphism. This suggests a Reidemeister relation $\sim_{i}$ on $\pi_{1}(X)$ defined by

$$
\alpha \sim_{i} \beta \Leftrightarrow \exists \gamma \in S_{i}: \alpha=\gamma \beta \phi_{i}\left(\gamma^{-1}\right) .
$$

Note that this is not an ordinary Reidemeister relation (or twisted conjugacy relation) since the homomorphism $\phi_{i}$ is not an endomorphism of $S_{i}$ (see the example below). Nevertheless, we will denote the number of equivalence classes of $\sim_{i}$, determined by the homomorphism $\phi_{i}: S_{i} \rightarrow \pi_{1}(X)$, by $R\left(\phi_{i}\right)$. The computation above shows that $\alpha \bar{f}_{i_{k}}^{*} \sim_{l f} \beta \bar{f}_{i_{k}}^{*}$ if and only if $\alpha \sim_{i_{k}} \beta$. We conclude that

$$
R(f)=R_{1}(f)+R_{2}(f)+\cdots+R_{r}(f)=R\left(\phi_{i_{1}}\right)+R\left(\phi_{i_{2}}\right)+\cdots+R\left(\phi_{i_{r}}\right) .
$$

If we denote the equivalence class of $\gamma \in \pi_{1}(X)$ for the relation $\sim_{i_{k}}$ by $[\gamma]_{i_{k}}$, then we have

$$
\operatorname{Fix}(f)=\bigcup_{k=1}^{r} \bigcup_{[\gamma]_{i_{k}}} p \operatorname{Fix}\left(\gamma \bar{f}_{i_{k}}^{*}\right)
$$

where the last union is taken over all $[\gamma]_{i_{k}}$ with $\gamma \in \pi_{1}(X)$. Note that this union is a disjoint union and some sets may be empty.

Applying this to the example, we choose representatives $\bar{f}_{1}^{*}$ and $\bar{f}_{3}^{*}$ for the $\sigma$-classes (so $i_{1}=1$ and $i_{2}=3$ ). For the sets $S_{i_{k}}$, we have $S_{1}=\left\{\left(z_{1}, z_{2}\right) \in \mathbb{Z}^{2} \mid z_{1}\right.$ is even $\}$ and $S_{3}=\mathbb{Z}^{2}$. Now $\phi_{1}: S_{1} \rightarrow \mathbb{Z}^{2}$ is defined by $\phi_{1}\left(z_{1}, z_{2}\right)=\left(\frac{z_{1}}{2},-z_{2}\right)$ and $\phi_{3}: S_{3} \rightarrow \mathbb{Z}^{2}$ 
by $\phi_{3}\left(z_{1}, z_{2}\right)=\left(-z_{1},-z_{2}\right)$. We compute $R_{1}(f)$ :

$$
\begin{aligned}
& \left(k_{1}, k_{2}\right) \bar{f}_{1}^{*} \sim_{l f}\left(l_{1}, l_{2}\right) \bar{f}_{1}^{*} \\
& \Longleftrightarrow\left(k_{1}, k_{2}\right) \sim_{1}\left(l_{1}, l_{2}\right) \\
& \Longleftrightarrow \exists\left(z_{1}, z_{2}\right) \in S_{1}:\left(k_{1}, k_{2}\right)=\left(z_{1}, z_{2}\right)\left(l_{1}, l_{2}\right) \phi_{1}\left(-z_{1},-z_{2}\right) \\
& \Longleftrightarrow \exists\left(z_{1}, z_{2}\right) \in S_{1}:\left\{\begin{array}{l}
k_{1}=z_{1}+l_{1}-\frac{z_{1}}{2} \\
k_{2}=z_{2}+l_{2}+z_{2}
\end{array}\right. \\
& \Longleftrightarrow \exists\left(z_{1}, z_{2}\right) \in S_{1}:\left\{\begin{array}{l}
2\left(k_{1}-l_{1}\right)=z_{1} \\
\frac{k_{2}-l_{2}}{2}=z_{2}
\end{array}\right. \\
& \Longleftrightarrow k_{2} \text { and } l_{2} \text { have the same parity }
\end{aligned}
$$

This means that there are two equivalence classes so $R_{1}(f)=2$. In terms of fixed point classes, the lift-factors $\alpha \bar{f}_{1}^{*}$ (and also the lift-factors $\left.\alpha \bar{f}_{2}^{*}\right)$ give rise to two fixed point classes.

Analogously, for the computation of $R_{2}(f)$ we find that $\left(k_{1}, k_{2}\right) \bar{f}_{3}^{*} \sim_{l f}$ $\left(l_{1}, l_{2}\right) \bar{f}_{3}^{*}$ if and only if there exists $\left(z_{1}, z_{2}\right) \in \mathbb{Z}^{2}$ with $z_{1}=\frac{k_{1}-l_{1}}{2}$ and $z_{2}=\frac{k_{2}-l_{2}}{2}$, which means that both $k_{1}$ and $l_{1}$, and $k_{2}$ and $l_{2}$ have the same parity. Thus there are four equivalence classes, so $R_{2}(f)=4$, which means that the lift-factors $\alpha \bar{f}_{3}^{*}$ give rise to four fixed point classes.

We conclude that

$$
R(f)=R_{1}(f)+R_{2}(f)=R\left(\phi_{1}\right)+R\left(\phi_{3}\right)=2+4=6,
$$

so $N(f) \leq 6$.

Computing the fixed point classes explicitly leads to two fixed point classes

$$
p \operatorname{Fix}\left((0,0) \bar{f}_{1}^{*}\right)=\{p(0,0)\} \text { and } p \operatorname{Fix}\left((0,1) \bar{f}_{1}^{*}\right)=\left\{p\left(0, \frac{1}{2}\right)\right\}
$$

for the $\sigma$-class $\left\{\bar{f}_{1}^{*}, \bar{f}_{2}^{*}\right\}$ and four fixed point classes

$$
\begin{gathered}
p \operatorname{Fix}\left((0,0) \bar{f}_{3}^{*}\right)=\left\{p\left(0, \frac{1}{4}\right)\right\}, p \operatorname{Fix}\left((0,1) \bar{f}_{3}^{*}\right)=\left\{p\left(0, \frac{3}{4}\right)\right\}, \\
p \operatorname{Fix}\left((1,0) \bar{f}_{3}^{*}\right)=\left\{p\left(\frac{1}{2}, \frac{1}{4}\right)\right\} \text { and } p \operatorname{Fix}\left((1,1) \bar{f}_{3}^{*}\right)=\left\{p\left(\frac{1}{2}, \frac{3}{4}\right)\right\}
\end{gathered}
$$

for the $\sigma$-class $\left\{\bar{f}_{3}^{*}\right\}$. So $f$ has in total six fixed point classes, each class is a singleton.

Because the covering map is a local homeomorphism, the fixed point index of $f$ at each of these points will agree with the fixed point index of the lifts $\bar{f}_{i}^{*}$ at their fixed points. Since these lifts are linear maps with isolated fixed points, each of these indices will be +1 or -1 . Thus each of the fixed point classes of $f$ is essential, and so $N(f)=6$. 


\section{The Circle}

As before we represent $S^{1}$ as the complex numbers of norm one. We define $f: S^{1} \rightarrow D_{2}\left(S^{1}\right)$ by letting $f(z)$ be the two square roots of $z$. Then the $\operatorname{map}\left(\bar{f}_{1}^{*}, \bar{f}_{2}^{*}\right): \mathbb{R} \rightarrow F_{2}(\mathbb{R}, \mathbb{Z})$ defined by $\bar{f}_{1}^{*}(t)=\frac{t}{2}$ and $\bar{f}_{2}^{*}(t)=\frac{t+1}{2}=\bar{f}_{1}^{*}(t)+\frac{1}{2}$ is a lifting of $f$, which we choose as the basic lifting. Since the covering transformations are the elements $k \in \mathbb{Z}$ acting on $\mathbb{R}$ by translations $t \mapsto k+t$, then

$$
\bar{f}_{i}^{*}(k+t)=\frac{k}{2}+\bar{f}_{i}^{*}(t)
$$

for $i=1,2$.

Thus if $k$ is even, then $\bar{f}_{i}^{*}(k+t)=\frac{k}{2}+\bar{f}_{i}^{*}(t)$, with $\frac{k}{2} \in \mathbb{Z}$. If $k$ is odd, then

$$
\bar{f}_{1}^{*}(k+t)=\frac{k}{2}+\bar{f}_{1}^{*}(t)=\frac{k-1}{2}+\frac{1}{2}+\bar{f}_{1}^{*}(t)=\frac{k-1}{2}+\bar{f}_{2}^{*}(t)
$$

and

$$
\bar{f}_{2}^{*}(k+t)=\frac{k}{2}+\bar{f}_{2}^{*}(t)=\frac{k+1}{2}-\frac{1}{2}+\bar{f}_{2}^{*}(t)=\frac{k+1}{2}+\bar{f}_{1}^{*}(t),
$$

with $\frac{k \pm 1}{2} \in \mathbb{Z}$. Therefore, $\sigma_{k}$ is the identity permutation of $\{1,2\}$ if $k$ is even and the other permutation if $k$ is odd. The functions $\phi_{i}: \mathbb{Z} \rightarrow \mathbb{Z}$ are defined by $\phi_{1}(k)=\frac{k}{2}$ if $k$ is even and $\phi_{1}(k)=\frac{k-1}{2}$ if $k$ is odd and $\phi_{2}(k)=\frac{k}{2}$ if $k$ is even and $\phi_{2}(k)=\frac{k+1}{2}$ if $k$ is odd. In this case it is easy to see that the $\phi_{i}$ are not homomorphisms; for instance $\phi_{1}(1)=0$ but $\phi_{1}(1+1)=1$.

We will show that $R(f)=1$. That is, for $\alpha, \beta \in \mathbb{Z}$ and $i, j \in$ $\{1,2\}$, we always have $[(\alpha, i)]=[(\beta, j)]$ in the notation of Section 2 ,

In the sequel we will use the results from the previous section. As $\sigma_{1}(1)=2$, there is only one $\sigma$-class and therefore $R(f)=R_{1}(f)=$ $R\left(\phi_{i_{1}}\right)$. We choose $i_{1}=1$. It is easy to see that $S_{1}=2 \mathbb{Z}$. Recall that $R\left(\phi_{1}\right)$ is the number of equivalence classes of the relation $\sim_{1}$ on $\mathbb{Z}$ where

$$
\begin{aligned}
\forall k, l \in \mathbb{Z}: k \sim_{1} l & \Leftrightarrow \exists z \in 2 \mathbb{Z}: k=z+l-\phi_{1}(z) \\
& \Leftrightarrow \exists z \in 2 \mathbb{Z}: k=z+l-\frac{z}{2} \\
& \Leftrightarrow \exists u \in \mathbb{Z}: k=l+u .
\end{aligned}
$$

It follows that for all $k, l \in \mathbb{Z}$ we have $k \sim_{1} l$ and hence $R(f)=$ $R\left(\phi_{1}\right)=1$. 
More generally, define an $n$-valued map $f: S^{1} \rightarrow D_{n}\left(S^{1}\right)$ by letting $f(z)$ be the set of $n$-th roots of $z^{d}$ for an integer $d \neq n$. Thus $f=\phi_{n, d}$ in the notation of [2]. Define liftings $\bar{f}_{1}^{*}, \ldots, \bar{f}_{n}^{*}$ by setting

$$
\bar{f}_{j}^{*}(t)=\frac{d t+j-1}{n} .
$$

Take $\bar{f}^{*}=\left(\bar{f}_{1}^{*}, \bar{f}_{2}^{*}, \ldots, \bar{f}_{n}^{*}\right)$ as the basic lifting of $f$.

We will prove that $\alpha+\bar{f}_{i}^{*} \sim_{l f} \beta+\bar{f}_{j}^{*}($ that is $[(\alpha, i)]=[(\beta, j)])$ if and only if $d \alpha+i=d \beta+j \bmod |d-n|$ and therefore that $R(f)=|d-n|$.

To compute $\bar{f}_{j}^{*}(t+k)$, divide $d k$ by $n$ to obtain integers $q, r$ with $d k=q n+r$ and $0 \leq r<n$. Then we have:

$$
\begin{aligned}
\bar{f}_{j}^{*}(t+k) & =\frac{d t+q n+r+j-1}{n}=\frac{d t+r+j-1}{n}+q \\
& = \begin{cases}\bar{f}_{j+r}^{*}(t)+q & \text { if } j+r \leq n \\
\bar{f}_{j+r-n}^{*}(t)+q+1 & \text { if } j+r>n .\end{cases}
\end{aligned}
$$

Thus $\sigma_{k}^{-1}(j)$ is either $j+r$ or $j+r-n$. In particular, if $\sigma_{k}^{-1}(j) \geq j$, then $\sigma_{k}^{-1}(j)=j+r$. Of the two cases in the formula above, we will only need the case where $j+r \leq n$. In this case we have

$$
\sigma_{k}^{-1}(j)=j+r \quad \phi_{j}(k)=q .
$$

Therefore if $j+r \leq n$ we compute

$$
d\left(\phi_{j}(k)-k\right)=d \phi_{j}(k)-d k=d q-(q n+r)=(d-n) q-r .
$$

Now let $\alpha, \beta \in \mathbb{Z}$ and $i, j \in\{1, \ldots, n\}$. We will prove that $[(\alpha, i)]=$ $[(\beta, j)]$ if and only if $d \alpha+i=d \beta+j \bmod |d-n|$. Assume that $[(\alpha, i)]=[(\beta, j)]$. Since the Reidemeister relation is symmetric, we may assume that $i \geq j$. Then there is some $k \in \mathbb{Z}$ with $\sigma_{k}^{-1}(j)=i$ and $\alpha=-k+\beta+\phi_{j}(k)$ (see Theorem 2.6 with $\gamma=-k$ ). As above, divide $d k$ by $n$ to obtain $d k=q n+r$. Since $i=\sigma_{k}^{-1}(j) \geq j$, we have $i=j+r$. Since $j+r=i \leq n$ then

$$
d(\alpha-\beta)=d\left(\phi_{j}(k)-k\right)=(d-n) q-r=(d-n) q-(i-j)
$$

and thus $d \alpha+i=d \beta+j \bmod |d-n|$.

For the converse, assume that $d \alpha+i=d \beta+j \bmod |d-n|$ where $\alpha, \beta \in \mathbb{Z}$ and $i, j \in\{1, \ldots, n\}$ with $i \geq j$. Then there is some $q \in \mathbb{Z}$ such that $d(\alpha-\beta)=(d-n) q+(j-i)$. Let $r=i-j$, and we have $d \alpha-d \beta=d q-n q-r$ so $n q+r$ is a multiple of $d$ and therefore there exists $k \in \mathbb{Z}$ such that $d k=n q+r$. We have $j+r=i \leq n$ so

$$
d(\alpha-\beta)=(d-n) q-r=d\left(\phi_{j}(k)-k\right)
$$


which means that $\alpha-\beta=\phi_{j}(k)-k$ so $[(\alpha, i)]=[(\beta, j)]$ if $d \neq 0$. In case $d=0$, it is easy to verify that $\alpha+\bar{f}_{i}^{*} \sim_{l f} \beta+\bar{f}_{j}^{*}$ if and only if $i=j$ so if and only if $d \alpha+i=d \beta+j \bmod |d-n|$.

We have proved that $[(\alpha, i)]=[(\beta, j)]$ if and only if $d \alpha+i=d \beta+j$ mod $|d-n|$ so there are $|d-n|$ Reidemeister classes characterized by the remainder of $d \alpha+i \bmod |d-n|$ and thus $R(f)=|d-n|$. The Reidemeister number is a homotopy invariant by Theorem 2.7. By Theorem 3.1 of [2], every $n$-valued self-map $f: S^{1} \rightarrow D_{n}\left(S^{1}\right)$ is homotopic to $\phi_{n, d}$ for some integer $d$, and this $d$ is called the degree of $f$. We have shown:

Theorem 5.1. Let $f: S^{1} \rightarrow D_{n}\left(S^{1}\right)$ be an n-valued map of degree $d \neq n$. Then $R(f)=|d-n|$.

We can also compute $R(f)$ in the case when $n=d$ :

Theorem 5.2. Let $f: S^{1} \rightarrow D_{n}\left(S^{1}\right)$ be the $n$-valued map of degree $n$. Then $R(f)=\infty$.

Proof. We use basic liftings of $f$ as above, letting $d=n$ :

$$
\bar{f}_{j}^{*}(t)=\frac{n t+j-1}{n}=t+\frac{j-1}{n} .
$$

For some $k \in \mathbb{Z}$, we will have $\bar{f}_{j}^{*}(t+k)=t+k+\frac{j-1}{n}=k+\bar{f}_{j}^{*}(t)$, and so $\sigma_{k}(j)=j$ for each $j$, and $\phi_{j}(k)=k$ for each $k$. Thus by Theorem 2.6 we have $\alpha+\bar{f}_{j}^{*} \sim_{l f} \beta+\bar{f}_{i}^{*}$ if and only if $i=j$ and $\alpha=\beta$. Since $\alpha$ and $\beta$ can be any integers, there are infinitely many lift-factor classes, and thus $R(f)=\infty$.

\section{The Orbit Configuration Space and the Uni- versal Cover}

The theory of lifting classes, Reidemeister classes, and twisted conjugacy of a single-valued map $f: X \rightarrow X$ is defined in terms of the universal cover $\tilde{X}$ of $X$ and the induced homomorphism $f_{\#}$ : $\pi_{1}(X) \rightarrow \pi_{1}(X)$. The most direct generalization to an $n$-valued map $f: X \rightarrow D_{n}(X)$ would seem to involve the universal cover of $D_{n}(X)$ and the induced homomorphism $f_{\#}: \pi_{1}(X) \rightarrow \pi_{1}\left(D_{n}(X)\right)$. In this section, we discuss these ideas and describe why we have instead opted in Section 2 to use the orbit configuration space. We will show that for manifolds of dimension at least 3 , the two approaches are algebraically the same. In dimensions 1 and 2 (as long as $X$ is not the circle), the orbit configuration space approach is a 
quotient of the universal cover approach, but it still includes all the data necessary to compute the Nielsen theory of an $n$-valued map.

First we briefly review the Galois Correspondence for covering spaces (see [10, Theorem 1.38]) which states that there is a bijective correspondence between isomorphism classes of connected covering spaces over $X$ and conjugacy classes of subgroups of $\pi_{1}(X)$. We summarize the specific facts that we will need in a lemma:

Lemma 6.1. Let $A$ and $X$ be connected, locally connected, and semilocally simply connected spaces. Let $u: \tilde{A} \rightarrow A$ be the universal cover of $A$, and let $p: B \rightarrow A$ be some other normal connected cover.

a. There is a covering map $r: \tilde{A} \rightarrow B$ with $u=p \circ r$, and the covering group of $p: B \rightarrow A$ is $\pi_{1}(A) / N$ for some normal subgroup $N \leq \pi_{1}(A)$. If $N$ is trivial, then $r$ is a homeomorphism.

b. If $f: X \rightarrow A$ is a map and $\tilde{f}: \tilde{X} \rightarrow \tilde{A}$ is a lifting of $f$ to universal covers, then there is a lifting $\bar{f}: \tilde{X} \rightarrow B$ with $\bar{f}=r \circ \tilde{f}$.

c. If $f_{\#}: \pi_{1}(X) \rightarrow \pi_{1}(A)$ is the induced homomorphism of fundamental groups, then there is an induced homomorphism $\phi$ : $\pi_{1}(X) \rightarrow \pi_{1}(A) / N$ with $\phi=q \circ f_{\#}$, where $q$ is the canonical surjection $q: \pi_{1}(X) \rightarrow \pi_{1}(X) / N$.

We will apply the lemma above to the setting of maps $f: X \rightarrow$ $D_{n}(X)$, the covers $p^{n}: F_{n}(\tilde{X}, \pi) \rightarrow D_{n}(X)$ and the universal cover $u: \tilde{D}_{n}(X) \rightarrow D_{n}(X)$.

As a preliminary we must establish that $p^{n}: F_{n}(\tilde{X}, \pi) \rightarrow D_{n}(X)$ is a connected cover, and that $D_{n}(X)$ has the appropriate connectedness properties for covering space theory. We begin with the connectedness of $F_{n}(\tilde{X}, \pi)$. Our argument closely resembles a similar argument for $F_{n}(X)$. The idea is due to Farber, in [6, Section 8].

Farber's result is important in the topological theory of robot motion planning. The following result, when $X$ is a 1-complex other than the circle or interval, essentially says that any labeled set of $n$ robots moving along a track with junctions can be rearranged without colliding to move to any desired locations.

Lemma 6.2. Let $X$ be a connected polyhedron not homeomorphic to the interval or circle. Then $F_{n}(X)$ is path connected.

Proof. The fact that $X$ is not the interval or circle means that some subdivision of $X$ must have a vertex which meets at least 3 edges. 
Such a vertex is called an essential vertex in [6]. Let $v$ be an essential vertex of $X$, let $e$ be one of the edges meeting $v$, and let $z=\left(z_{1}, \ldots, z_{n}\right) \in F_{n}(X)$ be some ordered $n$-configuration with $z_{i} \in e$ for each $i$.

To show that $F_{n}(X)$ is connected, it suffices to show that any other ordered $n$-configuration $x=\left(x_{1}, \ldots, x_{n}\right) \in F_{n}(X)$ can be connected to $z$ by a path in $F_{n}(X)$. This path in $F_{n}(X)$ would consist of $n$ paths $\gamma_{i}$ connecting $x_{i}$ to $z_{i}$ such that $\gamma_{i}(t) \neq \gamma_{j}(t)$ for any $t$ and all $i \neq j$. We imagine such a set of paths as representing a continuous motion of the points $x_{i}$ to $z_{i}$ during which the $n$ points never collide.

It is clear that the points $\left\{x_{1}, \ldots, x_{n}\right\}$, if we disregard their ordering, can be moved without colliding into the points $\left\{z_{1}, \ldots, z_{n}\right\}$. To achieve this we proceed as follows. First of all, by further subdividing $X$ if needed we can assume that all the points $x_{i}$ are vertices of $X$. From now on, we replace $X$ by its 1 -skeleton and we will construct a path where the $x_{i}$ move along this 1-skeleton. We can now further delete edges of $X$, different from $e$ and two other edges meeting $v$, until we reach the situation in which $X$ is a tree. We now equip $X$ with the standard metric as a tree (in which every edge has length 1) and order the $x_{i}$ by increasing distance from $v$. Then we may move the points without colliding into the edge $e$ one at a time starting with those nearest to $v$. (Note this argument shows that $D_{n}(X)$ is connected for any connected polyhedron, even the circle or interval.)

To show that $F_{n}(X)$ is connected, it remains only to show that the configuration $\left(z_{1}, \ldots, z_{n}\right)$ can be moved without collision into any permutation of itself. This is accomplished by Farber's algorithm described in detail in [6] (a similar procedure is used in [15]). Briefly, the points may rearrange without colliding by using the essential vertex as a three-way road junction: Let $e_{2}$ and $e_{3}$ be two other edges meeting $v$. If for example $z_{3}$ and $z_{4}$ wish to exchange their positions, then first $z_{1}$ and $z_{2}$ can move into $e_{2}$. Then $z_{3}$ moves into $e_{3}$, then $z_{4}$ moves into $e_{2}$, then $z_{3}$ moves back into $e$ followed by $z_{4}$ and finally by $z_{2}$ and $z_{1}$. In this way any desired permutation of the $z_{i}$ can be achieved by noncolliding paths.

In the following result we generalize Farber's procedure to the setting of the orbit configuration space. There is a motion-planning interpretation of this theorem: if we have $n$ objects moving along tracks which are arranged like a covering space, for example vertically stacked tracks as in a parking garage, then any labeled set of $n$ robots moving along these tracks can be rearranged into any desired 
locations without ever colliding or moving directly above or below each other.

Theorem 6.3. Let $X$ be a connected polyhedron not homeomorphic to the interval or circle. Then $F_{n}(\tilde{X}, \pi)$ is path connected.

Proof. We will mimic the argument used in Lemma 6.2. Let $v$ be an essential vertex $X$ with some incident edge $e$, and choose $\tilde{v} \in \tilde{X}$ with $p(\tilde{v})=v$ and the edge $\tilde{e}$ incident at $\tilde{v}$ with $p(\tilde{e})=e$. Let $\left(\tilde{z}_{1}, \ldots, \tilde{z}_{n}\right) \in F_{n}(\tilde{X}, \pi)$ with $\tilde{z}_{i} \in \tilde{e}$ for each $i$, and we will show that any configuration $\left(\tilde{x}_{1}, \ldots, \tilde{x}_{n}\right) \in F_{n}(\tilde{X}, \pi)$ can be connected to $\left(\tilde{z}_{1}, \ldots, \tilde{z}_{n}\right)$ by a path in $F_{n}(\tilde{X}, \pi)$.

We imagine this path in $F_{n}(\tilde{X}, \pi)$ as representing a continuous motion of the points $\tilde{x}_{i}$ into the points $\tilde{z}_{i}$ such that the projections $x_{i}=p\left(\tilde{x}_{i}\right)$ never collide in $X$. By first lifting the path obtained in Lemma 6.2, we can move each of the points $\tilde{x}_{i}$ into some covering translations of $\tilde{e}$, reaching points $\gamma_{i} \tilde{z}_{i}$ for some $\gamma_{i} \in \pi_{1}(X)$ such that the projections are noncolliding during the motion.

It remains to show that the configuration $\left(\gamma_{1} \tilde{z}_{1}, \ldots, \gamma_{n} \tilde{z}_{n}\right)$ can be moved to $\left(\tilde{z}_{1}, \ldots, \tilde{z}_{n}\right)$ with noncolliding projections. Since we may achieve any permutation of the $\gamma_{i} z_{i}$ by moving points so that their projections move according to the three-way junction at $v$, it will be enough to show that $\left(\gamma_{1} \tilde{z}_{1}, \gamma_{2} \tilde{z}_{2}, \ldots, \gamma_{n} \tilde{z}_{n}\right)$ can be moved to $\left(\tilde{z}_{1}, \gamma_{2} \tilde{z}_{2}, \ldots, \gamma_{n} \tilde{z}_{n}\right)$ with noncolliding projections.

Viewing $\gamma_{1} \in \pi_{1}(X)$ as a loop provides a path from $\gamma_{1} \tilde{z}_{1}$ to $\tilde{z}_{1}$. Again using the three-way junction at $v$ we can move $\gamma_{1} \tilde{z}$ along this path to $\tilde{z}_{1}$ so that no collisions occur in the projection. (If a collision of projections is about to occur in $e$, the two points can use the three-way junction to exchange positions before continuing.) Thus $\left(\gamma_{1} \tilde{z}_{1}, \gamma_{2} \tilde{z}_{2}, \ldots, \gamma_{n} \tilde{z}_{n}\right)$ can be moved to $\left(\tilde{z}_{1}, \gamma_{2} \tilde{z}_{2}, \ldots, \gamma_{n} \tilde{z}_{n}\right)$ with noncolliding projections as desired.

In the case where $X$ is the interval or circle, $F_{n}(\tilde{X}, \pi)$ is indeed disconnected.

Example 6.4. Let $X$ be the interval $[0,1]$. Then $\tilde{X}=X$ and $\pi_{1}(X)$ is trivial, so $F_{n}(\tilde{X}, \pi)=F_{n}([0,1])$. We first consider the case $n=2$. It is clear that in this case

$$
F_{2}([0,1])=\left\{(x, y) \in[0,1]^{2} \mid x \neq y\right\},
$$

with the usual subspace topology from $\mathbb{R}^{2}$, is disconnected.

For $n>2$, there is a natural surjection $f: F_{n}([0,1]) \rightarrow F_{2}([0,1])$ which discards the last $n-2$ points. Since $F_{n}([0,1])$ has a disconnected image under $f$, it must itself be disconnected. 
Example 6.5. Let $X$ be the circle $S^{1}$. Then $\tilde{X}$ may be identified with the line $\mathbb{R}$, and we will show that $F_{n}(\mathbb{R}, \pi)$ is disconnected.

We again first consider the case $n=2$. In this case we have:

$$
F_{2}(\mathbb{R}, \pi)=\left\{(x, y) \in \mathbb{R}^{2} \mid x-y \notin \mathbb{Z}\right\},
$$

with the subspace topology from $\mathbb{R}^{2}$, and this is disconnected.

For $n>2$, a similar argument as in Example 6.4 shows that $F_{n}(\mathbb{R}, \pi)$ is also disconnected.

Now we show that $D_{n}(X)$ has the required connectedness properties for classical covering space theory.

Lemma 6.6. If $X$ is a connected finite polyhedron, then $D_{n}(X)$ is connected, locally path connected, and locally simply connected.

Proof. We have already shown in the proof of Lemma 6.2 that $D_{n}(X)$ is connected.

Since $F_{n}(X)$ is a finite covering of $D_{n}(X)$, any point of $D_{n}(X)$ has a open neighbourhood which is homeomorphic to an open set of $F_{n}(X)$. Therefore, it is enough to show that $F_{n}(X)$ is locally path connected and locally simply connected.

Since $X$ is a finite polyhedron, $X$ itself is locally path connected and locally simply connected.

As $X$ is a Hausdorff space, we have that for all $1 \leq i<j \leq n$, the set $K_{i, j}=\left\{\left(x_{1}, x_{2}, \ldots, x_{n}\right) \in X^{n} \mid x_{i}=x_{j}\right\}$ is a closed subset of $X^{n}$. It follows that

$$
F_{n}(X)=X^{n} \backslash\left(\bigcup_{1 \leq i<j \leq n} K_{i, j}\right)
$$

is an open subset of $X^{n}$.

Now, let $p=\left(x_{1}, x_{2}, \ldots, x_{n}\right)$ be any point of $F_{n}(X)$. As $F_{n}(X)$ is an open subset of $X^{n}$ and $X$ is locally path connected and locally simply connected, any neighbourhood of $p$ contains a subset $U=$ $U_{1} \times U_{2} \times \cdots U_{n} \subseteq F_{n}(X)$ where each $U_{i}$ is path connected and simply connected. It follows that $U$ itself is path connected and simply connected, and therefore $F_{n}(X)$ is locally path connected and locally simply connected, which completes the proof.

Now we return to the discussion of the relationship between $F_{n}(\tilde{X}, \pi)$ and the universal cover $u: \tilde{D}_{n}(X) \rightarrow D_{n}(X)$. The group of covering transformations is isomorphic to $\pi_{1}\left(D_{n}(X)\right)$, which is a well studied object: it is the full braid group $B_{n}(X)$. 
When $F_{n}(\tilde{X}, \pi)$ is connected, we have a connected covering $p^{n}$ : $F_{n}(\tilde{X}, \pi) \rightarrow D_{n}(X)$. Thus by Lemma 6.1 (国) there is a covering $\operatorname{map} r: \tilde{D}_{n}(X) \rightarrow F_{n}(X, \pi)$ with $u=p^{n} \circ r$ :

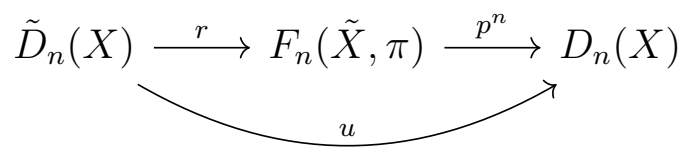

Since the covering group of $F_{n}(\tilde{X}, \pi)$ is $\pi_{1}(X)^{n} \rtimes \Sigma_{n}$, Lemma 6.1 (b) shows that $\pi_{1}(X)^{n} \rtimes \Sigma_{n}$ occurs naturally as a quotient of $B_{n}(X)$ by some normal subgroup. By Lemma 6.1 (ㄷ) there is a homomorphism $\Phi_{f}: \pi_{1}(X) \rightarrow \pi_{1}(X)^{n} \rtimes \Sigma_{n}$ such that the following diagram commutes:

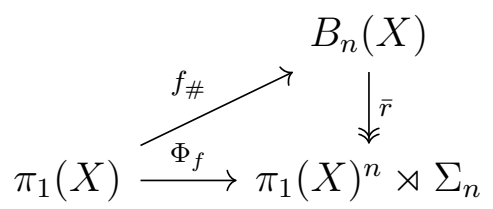

where the vertical arrow is the quotient homomorphism induced by the covering map $r$.

In terms of the orbit configuration space, this homomorphism $\Phi_{f}$ plays the same role that the induced homomorphism $f_{\#}$ plays in terms of the universal covering space. In fact we have already made use of $\Phi_{f}$ in the previous sections since it agrees with the morphism $\psi_{f}$ we introduced in Section 2, as the next result demonstrates.

Theorem 6.7. Let $X$ be a polyhedron not homeomorphic to the circle or interval, and let $f: X \rightarrow D_{n}(X)$ be a map, then $\Phi_{f}$ is the morphism $\psi_{f}$.

Proof. Let $\bar{f}^{*}=\left(\bar{f}_{1}^{*}, \ldots, \bar{f}_{n}^{*}\right)$ be the basic lifting of $f$, and let $F$ : $\tilde{X} \rightarrow \tilde{D}_{n}(X)$ be a lifting of $f$ such that the diagram commutes:

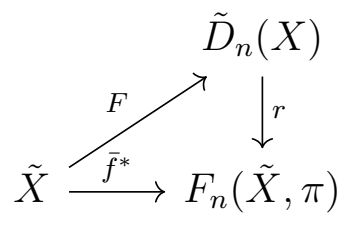

Since $f_{\#}$ is the induced homomorphism of $f$ on the fundamental group, we can choose base points in $\tilde{D}_{n}(X)$ so that, for any $\gamma \in$ $\pi_{1}(X)$ and $\tilde{x} \in \tilde{X}$, we have:

$$
F(\gamma \tilde{x})=f_{\#}(\gamma) F(\tilde{x})
$$


Applying $r$ to the above gives:

$$
\bar{f}^{*}(\gamma \tilde{x})=\Phi_{f}(\gamma) \bar{f}^{*}(\tilde{x}) .
$$

We also have by definition of $\psi_{f}$ that

$$
\bar{f}^{*}(\gamma \tilde{x})=\psi_{f}(\gamma) \bar{f}^{*}(\tilde{x}) .
$$

Since the action of $\pi_{1}(X)^{n} \rtimes \Sigma_{n}$ on $F_{n}(\tilde{X}, \pi)$ is a covering action, and $\Phi_{f}(\gamma) \bar{f}^{*}(\tilde{x})=\psi_{f}(\gamma) \bar{f}^{*}(\tilde{x})$, this means that $\Phi_{f}(\gamma)=\psi_{f}(\gamma)$ as desired.

In the case where $X$ is a manifold of dimension at least 3 , the algebra given by the universal covers and the orbit configuration space in diagram (2) are isomorphic:

Theorem 6.8. Let $X$ be a connected polyhedron which is a smooth manifold of dimension at least 3. Then $\pi_{1}\left(D_{n}(X)\right)=B_{n}(X)$ is isomorphic to $\pi_{1}(X)^{n} \rtimes \Sigma_{n}$.

Proof 2 We will use the following algebraic characterization of the semidirect product: If $H, G$, and $N$ are groups and we have a short exact sequence:

$$
1 \rightarrow H \stackrel{i}{\rightarrow} G \stackrel{j}{\rightarrow} N \rightarrow 1
$$

then $G \cong H \rtimes N$ if the sequence is right-split, i.e. there is a homomorphism $k: N \rightarrow G$ such that $j \circ k$ is the identity on $N$.

Let $P_{n}(X) \subseteq B_{n}(X)$ be the subgroup of "pure braids", those for which the underlying permutation of the strands is trivial. There is a well-known short exact sequence (9], page 16):

$$
1 \rightarrow P_{n}(X) \hookrightarrow B_{n}(X) \stackrel{j}{\rightarrow} \Sigma_{n} \rightarrow 1,
$$

where the map from $P_{n}(X)$ to $B_{n}(X)$ is the inclusion, and $j$ is the underlying permutation of the braid.

It is a classical theorem of Birman [1, Theorem 1] that when $X$ is a smooth manifold of dimension 3 or higher, the pure braid group $P_{n}(X)$ is isomorphic to $\pi_{1}(X)^{n}$. (Intuitively: since $X$ has high enough dimension, braid strands can pass through each other and so there is no classical braiding of strands wrapping around one another. The only nontrivial elements of $P_{n}(X)$ arise when the $n$ strands wrap around holes in the space.) Thus our short exact sequence becomes:

$$
1 \rightarrow \pi_{1}(X)^{n} \hookrightarrow B_{n}(X) \stackrel{j}{\rightarrow} \Sigma_{n} \rightarrow 1,
$$

\footnotetext{
${ }^{2}$ The authors thank Daciberg Gonçalves for suggesting this proof.
} 
and we need only find a right-inverse $k: \Sigma_{n} \rightarrow B_{n}(X)$ of $j$.

Since $X$ is a manifold of dimension 3 or higher, let $U \subset X$ be an open set which is homeomorphic to an open $m$-ball, $m \geq 3$. Let $i$ : $D_{n}(U) \rightarrow D_{n}(X)$ be the inclusion, which induces a homomorphism on fundamental groups $i_{\#}: B_{n}(U) \rightarrow B_{n}(X)$. Birman's result shows that $P_{n}(U) \cong \pi_{1}(U)^{n} \cong\{1\}$. Then the short exact sequence for $U$ takes the form:

$$
1 \rightarrow 1 \hookrightarrow B_{n}(U) \rightarrow \Sigma_{n} \rightarrow 1
$$

and therefore $B_{n}(U) \cong \Sigma_{n}$. Under these isomorphisms, $i_{\#}$ gives us a homomorphism $k: \Sigma_{n} \rightarrow B_{n}(X)$.

It remains only to show that $k$ is a right-inverse of $j$, but this is clear: if we begin with a permutation $\sigma \in \Sigma_{n}$, then $k(\sigma) \in B_{n}(X)$ is a braid in $X$ which is induced by inclusion from a braid in $U$ with underlying permutation $\sigma$. Thus the underlying permutation of $k(\sigma)$ is $\sigma$, which is to say that $j(k(\sigma))=\sigma$ as desired.

\section{The Jiang subgroup}

Let $f: X \rightarrow D_{n}(X)$ be an $n$-valued map. A homotopy $H: X \times$ $I \rightarrow D_{n}(X)$ is a cyclic homotopy of $f$ if $H(x, 0)=H(x, 1)=f(x)$ for all $x \in X$. A cyclic homotopy of $f$ will lift to a homotopy starting at the basic lifting $\bar{f}^{*}=\left(\bar{f}_{1}^{*}, \ldots, \bar{f}_{n}^{*}\right): \tilde{X} \rightarrow F_{n}(\tilde{X}, \pi)$ and ending at $\left(\gamma_{1} \bar{f}_{\sigma^{-1}(1)}^{*}, \ldots, \gamma_{n} \bar{f}_{\sigma^{-1}(n)}^{*}\right)$, where $\sigma$ is a permutation and $\gamma_{i} \in \pi_{1}(X)$. In this way, from each cyclic homotopy we obtain an element $\left(\gamma_{1}, \ldots, \gamma_{n} ; \sigma\right) \in \pi_{1}(X)^{n} \rtimes \Sigma_{n}$. The Jiang subgroup for $n$ valued maps $J_{n}\left(\bar{f}^{*}\right) \subseteq \pi_{1}(X)^{n} \rtimes \Sigma_{n}$ is the set of all such elements. For $n=1$, this definition is the same as that of the $\operatorname{subgroup} J(\tilde{f})$ of $\pi_{1}(X)$ introduced by Jiang; see [12], page 303

Proposition 7.1. The set $J_{n}\left(\bar{f}^{*}\right)$ is a subgroup of $\pi_{1}(X)^{n} \rtimes \Sigma_{n}$.

Proof. Let $\left(\alpha_{1}, \ldots, \alpha_{n} ; \eta\right),\left(\beta_{1}, \ldots, \beta_{n} ; \theta\right) \in J_{n}\left(\bar{f}^{*}\right)$. We will show that $J_{n}\left(\bar{f}^{*}\right)$ is a subgroup by proving that

$$
\left(\alpha_{1}, \ldots, \alpha_{n} ; \eta\right)\left(\beta_{1}, \ldots, \beta_{n} ; \theta\right)^{-1} \in J_{n}\left(\bar{f}^{*}\right) .
$$

Since $\left(\alpha_{1}, \ldots, \alpha_{n} ; \eta\right) \in J_{n}\left(\bar{f}^{*}\right)$, there is a cyclic homotopy $H$ of $f$ lifting to $n$ homotopies, of the $\bar{f}_{i}^{*}$ to $\alpha_{i} \bar{f}_{\eta^{-1}(i)}$. Similarly there is a cyclic homotopy $K$ of $f$ lifting to $n$ homotopies of $\bar{f}_{i}^{*}$ to $\beta_{i} \bar{f}_{\theta^{-1}(i)}^{*}$. Equivalently, $K$ lifts to homotopies of $\beta_{\theta(i)}^{-1} \bar{f}_{\theta(i)}^{*}$ to $\bar{f}_{i}^{*}$. Replacing $i$ by

\footnotetext{
${ }^{3}$ There is an extension of the Jiang subgroup, due to Gonçalves [7, that applies to maps $f: X \rightarrow Y$ and is quite different from $J_{n}(\bar{f})$, which concerns only $n$-valued maps, that is, the case $Y=D_{n}(X)$.
} 
$\eta^{-1}(i)$, we see that $K$ lifts to homotopies of $\beta_{\theta \eta^{-1}(i)}^{-1} \bar{f}_{\theta \eta^{-1}(i)}^{*}$ to $\bar{f}_{\eta^{-1}(i)}$. Then the concatenated homotopy $H * K^{-1}$, where $K^{-1}$ denotes the reverse of $K$, is a cyclic homotopy of $f$ lifting to homotopies of $\bar{f}_{i}^{*}$ to $\alpha_{i} \beta_{\theta \eta^{-1}(i)}^{-1} \bar{f}_{\theta \eta^{-1}(i)}^{*}$. Thus

$$
\left(\alpha_{1} \beta_{\theta \circ \eta^{-1}(1)}^{-1}, \ldots, \alpha_{n} \beta_{\theta \circ \eta^{-1}(n)}^{-1} ; \eta \circ \theta^{-1}\right) \in J_{n}(f),
$$

and this element is equal to $\left(\alpha_{1}, \ldots, \alpha_{n} ; \eta\right)\left(\beta_{1}, \ldots, \beta_{n} ; \theta\right)^{-1}$.

It is natural to ask how the subgroup $J_{n}\left(\bar{f}^{*}\right)$ depends on the choice of lifting $\bar{f}^{*}$. An alternative choice will change the Jiang subgroup, but in a predictable way. Any other lifting has the form $\Gamma \bar{f}^{*}$ for some $\Gamma \in \pi_{1}(X)^{n} \rtimes \Sigma_{n}$, as follows.

Theorem 7.2. Let $f: X \rightarrow D_{n}(X)$ be an $n$-valued map, and $\bar{f}^{*}$ : $\tilde{X} \rightarrow F_{n}(\tilde{X}, \pi)$ its basic lifting. Then $J_{n}\left(\Gamma \bar{f}^{*}\right)$ is isomorphic to $J_{n}\left(\bar{f}^{*}\right)$ by an inner automorphism of $\pi_{1}(X)^{n} \rtimes \Sigma_{n}$. In particular $J_{n}\left(\Gamma \bar{f}^{*}\right)=J_{n}\left(\bar{f}^{*}\right)^{\Gamma}$, where the exponent denotes conjugation by $\Gamma$.

Proof. We will show that for each $A=\left(\alpha_{1}, \ldots, \alpha_{n} ; \eta\right) \in J_{n}\left(\bar{f}^{*}\right)$, we have $\Gamma A \Gamma^{-1} \in J_{n}\left(\Gamma \bar{f}^{*}\right)$. Since $A \in J_{n}\left(\bar{f}^{*}\right)$, there is a cyclic homotopy of $f$ which lifts to a homotopy of $\bar{f}^{*}$ to $A \bar{f}^{*}$. This same cyclic homotopy, when lifted to start at $\Gamma \bar{f}^{*}$, will give a homotopy of $\Gamma \bar{f}^{*}$ to $\Gamma A \bar{f}^{*}=\left(\Gamma A \Gamma^{-1}\right) \Gamma \bar{f}^{*}$, and thus $\Gamma A \Gamma^{-1} \in J_{n}\left(\Gamma \bar{f}^{*}\right)$.

Recall from Section 2 that the homomorphism $\psi_{f}: \pi_{1}(X) \rightarrow$ $\pi_{1}(X)^{n} \rtimes \Sigma_{n}$ is defined by the requirement that $\forall \gamma \in \pi_{1}(X)$ : $\psi_{f}(\gamma) \bar{f}^{*}=\bar{f}^{*} \gamma$. ¿From the definition of $J_{n}\left(\bar{f}^{*}\right)$, we immediately obtain:

Theorem 7.3. Let $f: X \rightarrow D_{n}(X)$, and let $\bar{f}^{*}: \tilde{X} \rightarrow F_{n}(\tilde{X}, \pi)$ be the basic lifting. Then there is a cyclic homotopy from $\bar{f}^{*}$ to $\bar{f}^{*} \gamma$ for every $\gamma \in \pi_{1}(X)$ if and only if $\psi_{f}\left(\pi_{1}(X)\right) \subseteq J_{n}\left(\bar{f}^{*}\right)$.

The condition that $\psi_{f}\left(\pi_{1}(X)\right) \subseteq J_{n}\left(\bar{f}^{*}\right)$ is the $n$-valued analogue of the condition in single-valued Nielsen theory that $f_{\#}\left(\pi_{1}(X)\right) \subseteq$ $J(\tilde{f})$. In the single-valued theory, this condition implies that all fixed point classes have the same index, and this can be used to show in many cases that $N(f)=R(f)$. In the $n$-valued theory the result is that some, though perhaps not all, of the fixed point classes have the same index.

Lemma 7.4. Let $f: X \rightarrow D_{n}(X)$ be a map and $\bar{f}^{*}=\left(\bar{f}_{1}^{*}, \ldots, \bar{f}_{n}^{*}\right)$ be the basic lifting. If $\psi_{f}\left(\pi_{1}(X)\right) \subseteq J_{n}\left(\bar{f}^{*}\right)$, then $p \operatorname{Fix}\left(\gamma \bar{f}_{j}^{*}\right)$ and $p \operatorname{Fix}\left(\delta \bar{f}_{j}^{*}\right)$ have the same fixed point index for each $j$ and any $\gamma, \delta \in$ $\pi_{1}(X)$. 
Proof. It suffices to show that $p \operatorname{Fix}\left(\bar{f}_{i}^{*}\right)$ and $p \operatorname{Fix}\left(\gamma \bar{f}_{i}^{*}\right)$ have the same index for any $i$ and any $\gamma \in \pi_{1}(X)$. First observe that $\gamma \bar{f}_{i}^{*} \sim_{l f} \bar{f}_{i}^{*} \gamma$ since $\gamma \bar{f}_{i}^{*}=\gamma \bar{f}_{i}^{*} \gamma \gamma^{-1}$. Thus $p \operatorname{Fix}\left(\gamma \bar{f}_{i}^{*}\right)=p \operatorname{Fix}\left(\bar{f}_{i}^{*} \gamma\right)$ by Theorem 2.9. By Theorem [7.3. since $\psi_{f}\left(\pi_{1}(X)\right) \subseteq J_{n}\left(\bar{f}^{*}\right)$ we have a cyclic homotopy of $\bar{f}_{i}^{*}$ to $\bar{f}_{i}^{*} \gamma$. Thus, by the homotopy invariance of the fixed point index (Lemma 6.4 of [14]), we have

$$
\operatorname{ind}\left(f, p \operatorname{Fix}\left(\bar{f}_{i}^{*}\right)\right)=\operatorname{ind}\left(f, p \operatorname{Fix}\left(\bar{f}_{i}^{*} \gamma\right)\right)=\operatorname{ind}\left(f, p \operatorname{Fix}\left(\gamma \bar{f}_{i}^{*}\right)\right) .
$$

Recall from Section 4 the definition of $\sigma$-classes: these are the equivalence classes of the relation defined by

$$
i \sim j \Longleftrightarrow \exists \gamma \in \pi_{1}(X): \sigma_{\gamma}(i)=j
$$

Proposition 7.5. Let $f: X \rightarrow D_{n}(X)$ be a map and $\bar{f}^{*}=\left(\bar{f}_{1}^{*}, \ldots, \bar{f}_{n}^{*}\right)$ be the basic lifting. If $\psi_{f}\left(\pi_{1}(X)\right) \subseteq J_{n}\left(\bar{f}^{*}\right)$, and $i$ and $j$ are in the same $\sigma$-class, then $p \operatorname{Fix}\left(\gamma \bar{f}_{i}^{*}\right)$ and $p \operatorname{Fix}\left(\delta \bar{f}_{j}^{*}\right)$ have the same index for any $\gamma, \delta \in \pi_{1}(X)$. In particular, if there is only one $\sigma$-class, then all the fixed point classes of $f$ have the same index and therefore either $N(f)=0$ or $N(f)=R(f)$.

Proof. If $i$ and $j$ are in the same $\sigma$-class, then by Lemma 4.1 there exists $\beta \in \pi_{1}(X)$ such that $\gamma \bar{f}_{i}^{*} \sim_{l f} \beta \bar{f}_{j}^{*}$. By Theorem 2.9 , it follows that $p \operatorname{Fix}\left(\gamma \bar{f}_{i}^{*}\right)=p \operatorname{Fix}\left(\beta \bar{f}_{j}^{*}\right)$, and then by Lemma 7.4 we have:

$$
\begin{aligned}
\operatorname{ind}\left(f, p \operatorname{Fix}\left(\gamma \bar{f}_{i}^{*}\right)\right) & =\operatorname{ind}\left(f, p \operatorname{Fix}\left(\beta \bar{f}_{j}^{*}\right)\right) \\
& =\operatorname{ind}\left(f, p \operatorname{Fix}\left(\delta \bar{f}_{j}^{*}\right)\right)
\end{aligned}
$$

For $t=\left(t_{1}, \ldots, t_{q}\right) \in \mathbb{R}^{q}$, denote the universal covering space of the torus $T^{q}$ by $p^{q}: \mathbb{R}^{q} \rightarrow T^{q}$ where $p^{q}(t)=\left(p\left(t_{1}\right), \ldots, p\left(t_{q}\right)\right)$ for $p\left(t_{j}\right)=\exp \left(i 2 \pi t_{j}\right)$. The condition of Theorem 7.3 which implies equality of indices is always satisfied for maps on tori:

Theorem 7.6. Let $T^{q}$ denote the q-torus, and let $f: T^{q} \rightarrow D_{n}\left(T^{q}\right)$ be a map. Then $\psi_{f}\left(\pi_{1}\left(T^{q}\right)\right) \subseteq J_{n}\left(\bar{f}^{*}\right)$.

Proof. Let $a \in \mathbb{Z}^{q} \cong \pi_{1}\left(T^{q}\right)$. We will show that there is a cyclic homotopy of $f$ which lifts to a homotopy of $\bar{f}^{*}(t)$ to $\bar{f}^{*}(t+a)$. For $t \in \mathbb{R}^{q}$ and $s \in[0,1]$, define $\bar{H}(t, s)=\bar{f}^{*}(t+s a)$. Then $\bar{H}$ is a homotopy of $\bar{f}^{*}(t)$ to $\bar{f}^{*}(t+a)$, and each stage of the homotopy is $n$-valued because $\bar{f}^{*}(t)$ is $n$-valued. Let $H\left(p^{q}(t), s\right)=\left(p^{q}\right)^{n}(\bar{H}(t, s))$. Then we can compute:

$$
\begin{aligned}
H\left(p^{q}(t), 0\right) & =\left(p^{q}\right)^{n}(\bar{H}(t, 0))=\left(p^{q}\right)^{n} \bar{f}^{*}(t)=f\left(p^{q}(t)\right) \\
H\left(p^{q}(t), 1\right) & =\left(p^{q}\right)^{n}(\bar{H}(t, 1))=\left(p^{q}\right)^{n} \bar{f}^{*}(t+a) \\
& =f\left(p^{q}(t+a)\right)=f\left(p^{q}(t)\right)
\end{aligned}
$$


where the last equality holds because $a \in \mathbb{Z}^{q}$. Thus $H$ is a cyclic homotopy of $f$, which lifts to $\bar{H}$, a homotopy of $\bar{f}^{*}(t)$ to $\bar{f}^{*}(t+a)$.

To illustrate the previous results, we apply them to the following class of $n$-valued maps introduced in [4].

We recall that a $q \times q$ integer matrix $A$ induces a map $f_{A}: \mathbb{R}^{q} / \mathbb{Z}^{q}=$ $T^{q} \rightarrow T^{q}$ by

$$
f_{A}\left(p^{q}(t)\right)=p^{q}(A t)=\left(p\left(A_{1} \cdot t\right), \ldots, p\left(A_{q} \cdot t\right)\right),
$$

where $A_{j}$ is the $j$-th row of $A$, and that $f_{A}$ is called a linear self-map of $T^{q}$.

We define $x=\left(x_{1}, \ldots, x_{q}\right), y=\left(y_{1}, \ldots, y_{q}\right) \in \mathbb{R}^{q}$ to be congruent $\bmod n$, written $x \equiv y(n)$, if $x_{j}-y_{j}$ is divisible by $n$ for all $j=$ $1, \ldots, q$.

For $k \in \mathbb{Z}$, let $\mathbf{k}=(k, k, \ldots, k) \in \mathbb{Z}^{q}$. Define $f_{n, A}^{(k)}: \mathbb{R}^{q} \rightarrow T^{q}$ by

$$
f_{n, A}^{(k)}(t)=p^{q}\left(\frac{1}{n}(A t+\mathbf{k})\right) .
$$

Theorem 7.7. ([4], Theorem 3.1) $A q \times q$ integer matrix $A$ induces an n-valued map $f_{n, A}: T^{q} \rightarrow D_{n}\left(T^{q}\right)$ defined by

$$
f_{n, A}\left(p^{q}(t)\right)=\left\{f_{n, A}^{(1)}(t), \ldots, f_{n, A}^{(n)}(t)\right\}
$$

if and only if $A_{i} \equiv A_{j}(n)$ for all $i, j \in\{1, \ldots, q\}$.

Since $f_{1, A}=f_{A}: T^{q} \rightarrow T^{q}$, the maps $f_{n, A}$ are called linear $n$ valued self-maps of tori.

The $n$-valued map $f_{n, A}$ lifts to $\bar{f}_{n, A}^{*}=\left(\bar{f}_{1}^{*}, \ldots, \bar{f}_{n}^{*}\right): \mathbb{R}^{q} \rightarrow F_{n}\left(\mathbb{R}^{q}, \mathbb{Z}^{q}\right)$ where

$$
\bar{f}_{j}(t)^{*}=\frac{1}{n}(A t+\mathbf{j}) .
$$

Proposition 7.8. Let $A$ be a $q \times q$ integer matrix such that $A_{i} \equiv$ $A_{j}(n)$ for all $i, j \in\{1, \ldots, q\}$ and let $f_{n, A}: T^{q} \rightarrow D_{n}\left(T^{q}\right)$ be the corresponding linear $n$-valued self-map of $T^{q}$, then all the fixed point classes of $f_{n, A}$ have the same index and therefore $N\left(f_{n, A}\right)=0$ or $N\left(f_{n, A}\right)=R\left(f_{n, A}\right)$.

Proof. The map $f_{n, A}$ lifts to $\bar{f}_{n, A}^{*}=\left(\bar{f}_{1}^{*}, \ldots, \bar{f}_{n}^{*}\right): \mathbb{R}^{q} \rightarrow F_{n}\left(\mathbb{R}^{q}, \mathbb{Z}^{q}\right)$ where

$$
\bar{f}_{j}^{*}(t)=\frac{1}{n}(A t+\mathbf{j}) .
$$

Define $\bar{H}=\left(\bar{h}_{1}, \ldots, \bar{h}_{n}\right): \mathbb{R}^{q} \times I \rightarrow F_{n}\left(\mathbb{R}^{q}, \mathbb{Z}^{q}\right)$ by setting

$$
\bar{h}_{j}(t, s)=\frac{1}{n}(A t+\mathbf{j}+\mathbf{s}) \text {. }
$$


Then for each $j<n$, the coordinate $j$ of $\bar{H}$ gives a homotopy of $\bar{f}_{j}^{*}$ to $\bar{f}_{j+1}^{*}$. By projecting this homotopy, we have ind $\left(f_{n, A}, p \operatorname{Fix}\left(\bar{f}_{j}^{*}\right)\right)=$ $\operatorname{ind}\left(f_{n, A}, p \operatorname{Fix}\left(\bar{f}_{j+1}^{*}\right)\right)$ for each $j<n$.

Now let $p \operatorname{Fix}\left(\gamma \bar{f}_{j}^{*}\right)$ and $p \operatorname{Fix}\left(\delta \bar{f}_{k}^{*}\right)$ be any two fixed point classes. By concatenating the homotopies above, $p \operatorname{Fix}\left(\bar{f}_{j}^{*}\right)$ and $p \operatorname{Fix}\left(\bar{f}_{k}^{*}\right)$ have the same index. By Theorem 7.6 we have $\psi_{f_{n, A}}\left(\pi_{1}\left(T^{q}\right)\right) \subseteq$ $J_{n}\left(\bar{f}_{n, A}^{*}\right)$, and so we may apply Lemma 7.4. We obtain:

$$
\begin{aligned}
\operatorname{ind}\left(f_{n, A}, p \operatorname{Fix}\left(\gamma \bar{f}_{j}^{*}\right)\right) & =\operatorname{ind}\left(f_{n, A}, p \operatorname{Fix}\left(\bar{f}_{j}^{*}\right)\right) \\
& =\operatorname{ind}\left(f_{n, A}, p \operatorname{Fix}\left(\bar{f}_{k}^{*}\right)\right)=\operatorname{ind}\left(f_{n, A}, p \operatorname{Fix}\left(\delta \bar{f}_{k}^{*}\right)\right) .
\end{aligned}
$$

Therefore all the fixed point classes have the same index and we conclude that $N\left(f_{n, A}\right)=0$ or $N\left(f_{n, A}\right)=R\left(f_{n, A}\right)$.

The Nielsen number of $f_{n, A}$ was calculated in [5] to be $N\left(f_{n, A}\right)=$ $n\left|\operatorname{det}\left(E-\frac{1}{n} A\right)\right|$ where $E$ is the identity matrix, and thus, if $N\left(f_{n, A}\right)$ is non-zero, we can conclude that $R\left(f_{n, A}\right)=n\left|\operatorname{det}\left(E-\frac{1}{n} A\right)\right|$ also.

\section{Split maps}

An $n$-valued map $f: X \rightarrow D_{n}(X)$ is split if there exist single-valued maps $f_{1}, \ldots, f_{n}: X \rightarrow X$ such that $f(x)=\left\{f_{1}(x), \ldots, f_{n}(x)\right\}$ for all $x \in X$. We write $f=\left\{f_{1}, \ldots, f_{n}\right\}$. Schirmer proved ([14], Corollary 7.2) that if $f=\left\{f_{1}, \ldots, f_{n}\right\}$, is split, then the Nielsen number of the $n$-valued map is related to the Nielsen number for single-valued maps by

$$
N(f)=N\left(f_{1}\right)+\cdots+N\left(f_{n}\right) .
$$

Recall from Section 2 that the homomorphism $\psi_{f}: \pi_{1}(X) \rightarrow$ $\pi_{1}(X)^{n} \rtimes \Sigma_{n}$ is defined by the requirement that $\forall \gamma \in \pi_{1}(X)$ : $\psi_{f}(\gamma) \bar{f}^{*}=\bar{f}^{*} \gamma$, and that we write $\psi_{f}(\gamma)=\left(\phi_{1}(\gamma), \ldots, \phi_{n}(\gamma) ; \sigma_{\gamma}\right)$.

Theorem 8.1. Let $f=\left(f_{1}, \ldots, f_{n}\right)$ be a split $n$-valued map with basic lifting $\bar{f}^{*}=\left(\bar{f}_{1}^{*}, \ldots, \bar{f}_{n}^{*}\right)$ where $\bar{f}_{i}^{*}$ is a lifting of $f_{i}$. Then $\sigma_{\alpha}$ is the identity permutation for every $\alpha$, and each $\phi_{i}$ is the induced homomorphism $f_{i \pi}: \pi_{1}(X) \rightarrow \pi_{1}(X)$ of $f_{i}$.

Proof. Since $\bar{f}^{*} \alpha=\psi_{f}(\alpha) \bar{f}^{*}=\left(\phi_{1}(\alpha) \bar{f}_{\sigma_{\alpha}^{-1}(1)}^{*}, \ldots, \phi_{n}(\alpha) \bar{f}_{\sigma_{\alpha}^{-1}(n)}^{*}\right)$, we have $\bar{f}_{i}^{*} \alpha=\phi_{i}(\alpha) \bar{f}_{\sigma_{\alpha}^{-1}(i)}^{*}$ for all $i$. By single-valued covering space theory $\bar{f}_{i}^{*} \alpha=f_{i \pi}(\alpha) \bar{f}_{i}^{*}$, where $f_{i \pi}$ is the induced fundamental group homomorphism of $f_{i}$. It follows that $\phi_{i}(\alpha) \bar{f}_{\sigma_{\alpha}^{-1}(i)}^{*}=f_{i \pi}(\alpha) \bar{f}_{i}^{*}$, so $\sigma_{\alpha}(i)=i$ and $\phi_{i}=f_{i \pi}$. 
Since the permutations $\sigma_{\alpha}$ are all the identity in the split case, the characterization of the equivalence relation $\sim_{l f}$ in Theorem 2.6 can be simplified.

Corollary 8.2. Let $f=\left(f_{1}, \ldots, f_{n}\right)$ be a split $n$-valued map with basic lifting $\bar{f}^{*}$. Then $[(\alpha, i)]=[(\beta, j)]$ if and only if $i=j$ and there is some $\gamma$ such that $\alpha=\gamma \beta f_{i \pi}\left(\gamma^{-1}\right)$, where $f_{i \pi}$ is the induced fundamental group homomorphism of $f_{i \pi}$.

The above corollary means that when $f$ splits, no fixed point class $p \operatorname{Fix}\left(\alpha \bar{f}_{i}^{*}\right)$ can equal any fixed point class $p \operatorname{Fix}\left(\beta \bar{f}_{j}^{*}\right)$ when $i \neq j$, and when $i=j$ these fixed point classes are equal exactly when they are equal according to the classical theory of Reidemeister classes of a single-valued map.

Theorem 8.3. Let $f=\left(f_{1}, \ldots, f_{n}\right)$ be a split $n$-valued map. Then

$$
R(f)=R\left(f_{1}\right)+\cdots+R\left(f_{n}\right),
$$

where on the right side, $R$ denotes the classical Reidemeister number of a single-valued map.

Proof. We have already shown that when $f$ is split, we have $[(\alpha, i)]=$ $[(\beta, j)]$ if and only if $i=j$ and $\alpha$ and $\beta$ are classically Reidemeister equivalent by $\phi_{i}$, the induced homomorphism of $f_{i}$. Thus the number of Reidemeister classes of $f$ is the total of the number of Reidemeister classes of the various $f_{i}$.

\section{References}

[1] Birman, J., On braid groups, Communications on Pure and Applied Mathematics 22, 41 - 72 (1969).

[2] Brown, R., Fixed points of n-valued multimaps of the circle, Bull. Polish Acad. Sci. Math. 54, 153 - 162 (2006).

[3] Brown, R. and Gonçalves, D. On the topology of n-valued maps, Advances in Fixed Point Theory 8, 205 - 220 (2018).

[4] Brown, R. and Lin, J., Coincidences of projections and linear $n$-valued maps of tori, Topol. Appl. 157, 1990 - 1998 (2010).

[5] Crabb, M., Lefschetz indices for n-valued maps, J. Fixed Point Theory Appl. 17, 153 - 186 (2015).

[6] Farber, M., Collision free motion planning on graphs, in Algorithmic Foundations of Robotics VI, Erdmann, M. et al eds., Springer, 123 - 138 (2005). 
[7] Gonçalves, D., Coincidence theory, in Handbook of Topological Fixed Point Theory, R. F. Brown et al eds., Springer, 3 - 42 (2005).

[8] Gonçalves, D. and Guaschi, J., Fixed points of n-valued maps, the fixed point property and the case of surfaces - a braid approach, Indag. Math. 29, 91 - 124 (2018).

[9] Hansen, V., Braids and Coverings, London Mathematical Society, 1989.

[10] Hatcher, A, Algebraic Topology, Dover (2002).

[11] Jiang, B., Estimation of the Nielsen numbers, Chinese Math. Acta 5, 330 - 339 (1964).

[12] Jiang, B., Lectures on Nielsen Fixed Point Theory, Contemporary Math. 14 (1983).

[13] Jiang, B., A primer of Nielsen fixed point theory, in Handbook of Topological Fixed Point Theory, R. F. Brown et al eds., Springer, 617 - 646 (2005).

[14] Schirmer, H., An index and Nielsen number for n-valued multifunctions, Fund. Math. 121, 201 - 219 (1984).

[15] Staecker, P. C., Maps on graphs can be deformed to be coincidence free, Topological Methods in Nonlinear Analysis 37, 377 - 381, (2011).

[16] Xicoténcatl, M., Orbit configuration spaces, Contemporary Mathematics 621, 113 - 132 (2014). 